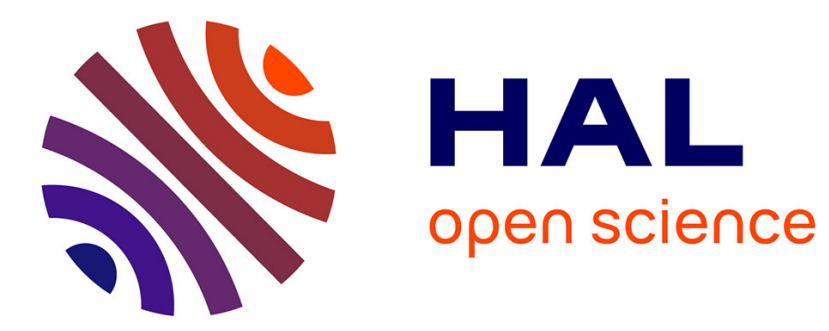

\title{
A Content-Based Active-Set Method for Pressure-Dependent Models of Water Distribution Systems with Flow Controls
}

\author{
Olivier Piller, Sylvan Elhay, Jochen Deuerlein, Angus Simpson
}

\section{To cite this version:}

Olivier Piller, Sylvan Elhay, Jochen Deuerlein, Angus Simpson. A Content-Based Active-Set Method for Pressure-Dependent Models of Water Distribution Systems with Flow Controls. Journal of Water Resources Planning and Management, 2020, 146 (4), 04020009, 13 pp. 10.1061/(ASCE)WR.19435452.0001160 . hal-02511724

\section{HAL Id: hal-02511724 \\ https://hal.science/hal-02511724}

Submitted on 19 Mar 2020

HAL is a multi-disciplinary open access archive for the deposit and dissemination of scientific research documents, whether they are published or not. The documents may come from teaching and research institutions in France or abroad, or from public or private research centers.
L'archive ouverte pluridisciplinaire $\mathbf{H A L}$, est destinée au dépôt et à la diffusion de documents scientifiques de niveau recherche, publiés ou non, émanant des établissements d'enseignement et de recherche français ou étrangers, des laboratoires publics ou privés. 
Author-produced version of the article published in Journal of Water Resources Planning and Management, 146(4), 04020009. The original publication is available at

https://doi.org/10.1061/(ASCE)WR.1943-5452.0001160. 


\title{
A Content-based Active-Set Method for Pressure-Dependent Models of Water Distribution Systems with Flow Controls
}

\author{
Olivier Piller ${ }^{1} \quad$ Sylvan Elhay ${ }^{2} \quad$ Jochen W. Deuerlein ${ }^{3}$ \\ Angus R. Simpson, M.ASCE ${ }^{4}$
}

January 22, 2020

\footnotetext{
${ }^{1}$ Senior Research Scientist, Irstea, Dept. of Water, Bordeaux Regional Centre, UR ETBX, 50 Ave. de Verdun, Gazinet, F-33612 Cestas, France \& Adjunct Senior Lecturer, School of Civil, Environmental and Mining Engineering, University of Adelaide, South Australia, 5005.

${ }^{2}$ Visiting Research Fellow, School of Computer Science, University of Adelaide, South Australia, 5005, sylvan.elhay@adelaide.edu.au. Corresponding author

${ }^{3}$ Senior Researcher, 3S Consult GmbH, Albtalstrasse 13, D 76137 Karlsruhe, Germany \& Adjunct Senior Lecturer, School of Civil, Environmental and Mining Engineering, University of Adelaide, South Australia, 5005 .

${ }^{4}$ Professor, School of Civil, Environmental and Mining Engineering, University of Adelaide, South Australia, 5005.
}

\begin{abstract}
In this paper a new method is proposed that solves for the steady state of pressure dependent models (PDMs) with flow control valves. Rather than model flow devices individually, the method solves the more general problem in which a water distribution system (WDS) has some link flows constrained to lie between upper and lower, or possibly equal, set limits. No heuristics are used to determine device states. The method is shown to be fast and its effectiveness is demonstrated on PDM WDSs with up to about 20,000 links and 18,000 nodes and 60 link flow constraints, some of which prescribe a fixed flow. deal with water distribution where there is insufficient supply.
\end{abstract}

\section{INTRODUCTION}

Hydraulic simulation solvers are used by water engineers in the design and management of large, complex, water distribution system (WDS) networks. Newton-type methods are frequently used to solve the non-linear model equations for the steady-state demand dependent 
model (DDM) link flows and nodal heads (Todini \& Rossman 2013) and very good methods now exist for pressure dependent model (PDM) WDSs in which there are no control devices (Deuerlein et al. 2019). However, real WDSs normally utilize control devices such as throttle control valves $(\mathrm{TCVs})$, check valves $(\mathrm{CHVs})$, flow control valves (FCVs), pressure sustaining valves (PSVs), pressure reducing valves (PRVs) and pumps but early methods developed for models which include some or all of these elements had considerable shortcomings. For example, iterative schemes for such models sometimes failed to converge or even converged to the wrong solution (Gorev et al. 2016). The status of PRVs, PSVs and FCVs was usually determined using heuristics: at each iteration the control devices are assigned states based on certain assumptions and if at the next iteration those assumptions no longer hold, changes of state are recorded for the devices. But even for very simple networks comprising just two regulating devices (e.g. one pressure regulating device and one flow regulating device) in series, hydraulic modelling software failed to converge to the correct solution for particular configurations and settings (see Simpson (1999) and Deuerlein et al. (2008)). More troubling is the fact that in most cases that produced a false solution, it was not immediately evident that a false solution had been produced. By comparison, Piller \& Bremond (2001) used a least-squares global optimization approach to determine the states of the PRVs in a system. Rather than the discrete control problem formulation, they solve the Karush-Kuhn-Tucker (KKT) equations for a constrained optimization problem.

FCVs restrict the flow through a valve to a certain preset maximum: a closed control loop is implemented consisting of flow measurement combined with a motor valve. From a modelling point of view, flow control devices can be classified into two categories: valves with fixed status (open, closed, fixed partly-opened state) and those whose hydraulic behavior or status is described by inequality conditions for the flow. Deuerlein et al. (2009) used content and co-content theory to characterize the conditions that ensure the existence and uniqueness of solutions in demand driven model (DDM) systems with flow control devices. They introduced a sub-differential analysis to deal with the non-differentiable flow versus head relationship that exists in WDSs with flow control devices and this led to a content-based, constrained optimization problem. The concept of content (which has the dimensions of power) was first introduced in the context of WDSs by Collins et al. (1978) and the content of a flow control device here is modelled in a way that is similar to that used previously for the content of a PDM node The flows range between a lower and upper bound in the same way that a pressure outflow relation (POR) describes how the nodal outflow is restricted to lie between zero and the nominal demand for a node according to the node's pressure. The head loss along a control device link is modelled by a nonlinear function of the flow (the given head loss function for pipes and a minor head loss function for devices). Thus, an FCV restricts the link flow to a preset 
maximum, $q_{\max }$, which is independent of the current difference between the heads of the link's initial and final nodes. If the flow is below $q_{\max }$, the valve is opened fully and behaves like a minor loss element. If conditions are such that the unrestricted flow would be higher than $q_{\text {max }}$, the head loss coefficient is increased (by reducing the opening of the valve until the preset flow level is reached again). In steady-state modelling this control behaviour can be modelled by a multivalued mapping like the one shown in Fig. 1. If the valve is in active control mode, it operates on the vertical line in Fig. 1. In this case the head difference between upstream and downstream nodes of the valve is composed of the head loss $h_{\text {set }}$ of the open valve for the maximum flow $q_{\max }$ and an additional minor head loss penalty which is incurred in restricting the flow to $q_{\max }$. In the mathematical model the additional minor head loss is represented by the Lagrange multiplier of the active flow constraint.

Piller \& van Zyl (2014) used external quadratic penalty function terms added to a valve's head loss equation to model FCVs. CHVs were modelled, taking into account the direction of flow, as FCVs with a minimum flow setting of zero. In this method the pressure control valves are handled by an optimization process which is external to the hydraulic solver and the method was shown to work well on DDM problems although it sometimes needed damping techniques or re-starting to achieve convergence. The authors did not apply this method to PDM problems although the method can be extended to these models.

Alvarruiz et al. (2015) modelled closed CHVs as zero flow pipes and FCVs as valves with flow at a preset level. Their scheme is based on the loop method, used the same heuristics as EPANET to determine device status and was applied only to DDM problems. In Alvarruiz et al. (2018) the authors improved on this work by using penalty methods (high resistance links).

In a later development, Deuerlein et al. (2019) presented a content-based active set method (ASM) for PDM problems without link flow constraints which is fast and reliable for a wide range of pressure outflow relations (PORs). The ASM solves an optimization problem with equality and inequality constraints. If in such optimizations an inequality constraint reaches equality at a point, the constraint is said to be active, binding or saturated at that point. ASMs get their name from the fact that in quadratic programming, apart from the feasibility requirement, the conditions for optimality involve only the set of active constraints, sometimes called the working set.

In another recent development Gorev et al. (2018) treated FCVs, PRVs, PSVs as links with adjustable resistance. Their focus was on a technique which extends EPANET 2 and as a consequence the hydraulic analysis of PDMs depended on adding to each demand node an artificial FCV, a fictitious node, an artificial CHV and an artificial source. The addition of this number of devices to large networks can increase the number of network components by 
percentages of thousands or hundreds of thousands. Searching the very much larger virtual network for valve states at each iteration in this approach presents a challenge to the method for large networks.

In this paper, the content function used in Deuerlein et al. (2019) forms the basis of a new ASM for PDM WDSs with FCVs and pumps. It was shown there that if a solution exists the flows are unique because of the strict convexity of the system content function. The range of models for which such solutions exist is greater than that for DDM problems by virtue of the relaxation of demand constraints in PDM modelling. There is no risk of isolated demands in this case since in PDM problems the nodal outflow can reduce to zero if the pressure is insufficient. In fact, for this model the non-existence of a solution can result only when the (wrong) choice of constraints for the flow control devices has the consequence that the polyhedral feasible set which is composed of (i) the continuity equation, (ii) the flow constraints of the control devices and (iii) the outflow conditions, is empty. Whether or not the feasible set is empty can be established using a linear program (LP) (see Boyd \& Vandenberghe (2009, 579) for details). It is shown that if 0 is included in all the constraint intervals then a solution always exists although it is only flows which are then determined uniquely and the heads may not be unique. The authors stress that the approach in this paper is somewhat more general than dealing with particular flow devices since it deals directly with constraints. Thus, the new method functions by including upper and lower flow bounds on links in the network. Assuming, without loss of generality, a positive flow direction, choosing upper flow bounds which fall below the link's unconstrained flow models an FCV while choosing a lower flow bound which is above the link's unconstrained flow models a pump. Using equal upper and lower link flow bounds, or setting link flow equality constraints (LFEC), amounts to prescribing, or fixing, a flow. The new method, referred to as an active set method for flow constraint (ASMFC) handles LFECs without difficulty.

The operation of the ASMFC is illustrated on a small example network and it's efficacy is demonstrated by applying it to eight case study networks with between 934 and 19,647 links and between 848 and 17,971 nodes. The case study networks each had 20, 40 or 60 cotree link flow constraints which either (i) limit the maximum flow in a link to $10 \%$ of its unconstrained value or (ii) prescribe a LFEC. The method determines, for various tested starting sets, the steadystate solutions, even on the largest case study network, in fewer than 14 iterations and with a termination test that uses the (smaller than would be used in practice) stopping tolerance of $10^{-10}$. The small stopping tolerance allows the quadratic convergence usually associated with Newton's method, which occurred in all cases, to be observed.

In summary, this paper presents a new method which solves for the heads, outflows and link flows of a WDS which models FCVs, pumps with flow control, check valves and closed 
valves. Pumps and FCVs are implemented as appropriate bounds on flow constraints. Fixed flows are introduced to model closed valves (zero flow equality constraints) and more complex controls while check valves are implemented as sign-constrained inequalities. It presents network managers with a convenient tool to study network decomposition. It establishes new conditions for the existence and uniqueness of solutions to the PDM FCV problem. The class of problems it solves is larger than the class of DDM problems which can be solved and extends the applicability of the method for DDM problems of Deuerlein et al. (2009) which used the loops method. The method does not use penalty functions which can frequently require more iterations than ASM methods.

The next section in the paper sets out some definitions and establishes some notation and the one following very briefly reviews the ASM PDM content model. The section following derives the new ASMFC and outlines some details that are necessary for the implementation of the practical algorithm: a set assignment algorithm, an initialization scheme and the handling of LFECs. The sections with examples are followed by some conclusions and suggestions for future work.

\section{DEFINITIONS AND NOTATION}

Consider a WDS whose network graph has $n_{p}$ links, or arcs, and $n_{j}+n_{f}$ nodes, or vertices: $n_{j}$ is the number of nodes at which the heads are unknown and $n_{f} \geq 1$ is the number of source nodes with fixed heads. The links of the network include control valves, pumps and pipes. Denote by $\boldsymbol{q}=\left(q_{1}, q_{2}, \ldots, q_{n_{p}}\right)^{T} \in \mathbb{R}^{n_{p}}$ the vector of unknown flows in the system, $\boldsymbol{h}=\left(h_{1}, h_{2}, \ldots, h_{n_{j}}\right)^{T} \in$ $\mathbb{R}^{n_{j}}$ the unknown heads at the nodes in the system, $\boldsymbol{u}=\left(u_{1}, u_{2}, \ldots, u_{n_{j}}\right)^{T} \in \mathbb{R}^{n_{j}}$ the vector of node elevations and $\boldsymbol{r}(\boldsymbol{q})=\left(r_{1}, r_{2}, \ldots, r_{n_{p}}\right)^{T}$ the vector of link resistance factors. Let $\boldsymbol{A}$ denote the $n_{p} \times n_{j}$, full rank, unknown-head arc-node incidence matrix, (ANIM): the $j i$ element of $\boldsymbol{A}$ is (i) -1 if node $i$ is at the end of $\operatorname{arc} j$, (ii) 0 if $\operatorname{arc} j$ does not connect to the node $i$, and (iii) 1 if arc $j$ starts at node $i$. Let $\boldsymbol{A}_{f}$ denote the ANIM, with a similar definition, for the fixed-head nodes. Let $\boldsymbol{h}^{0}$ denote the vector of elevations of the $n_{f}$ fixed-head nodes. Denote $\boldsymbol{a}=\boldsymbol{A}_{f} \boldsymbol{h}^{0}$. Denote by $\eta$ the exponent used in the head loss formula: $\eta=2$ for the Darcy-Weisbach model and $\eta=1.852$ for the Hazen-Williams model. Furthermore, denote by $\boldsymbol{G}(\boldsymbol{q}) \in \mathbb{R}^{n_{p} \times n_{p}}$ the diagonal matrix whose diagonal elements are defined as $[\boldsymbol{G}(\boldsymbol{q})]_{j j}=r_{j}\left|q_{j}\right|^{\eta-1}$. Then, $\boldsymbol{\xi}(\boldsymbol{q})=\boldsymbol{G}(\boldsymbol{q}) \boldsymbol{q}$ is the vector whose elements model the head losses of the links in the system. In general, (e.g. for the Darcy-Weisbach formula) $\boldsymbol{r}=\boldsymbol{r}(\boldsymbol{q})$ but for the Hazen-Williams formula $\boldsymbol{r}$ is independent of $\boldsymbol{q}$. Denote the vector of the nominal demands at the nodes with unknown-head by $\boldsymbol{d}=\left(d_{1}, d_{2}, \ldots, d_{n_{j}}\right)^{T} \in \mathbb{R}^{n_{j}}$. Denote by $\boldsymbol{c}(\boldsymbol{h}, \boldsymbol{d}) \in \mathbb{R}^{n_{j}}$ the vector whose elements are the POR function values at the $n_{j}$ nodes of the system. Throughout what follows, the symbol $\boldsymbol{O}$ denotes a zero matrix and $\boldsymbol{o}$ denotes a zero column vector of appropriate dimension for the 
particular case. The shorthand notation $\boldsymbol{x}+a$, where $\boldsymbol{x}$ is a vector and $a$ is a scalar, will be used to denote the case where every component of $\boldsymbol{x}$ has $a$ added to it. Furthermore, it will be assumed that any matrix inverses which are shown do exist.

Turning now to PDM problems, assume, for simplicity and without loss of generality, that every node has the same minimum pressure head, $h_{m}$, and the same service pressure head, $h_{s}$. Individualized minimum and service pressure heads can be implemented by replacing $h_{m}$ by $h_{m i}$ and $h_{s}$ by $h_{s i}$ throughout but presents no further difficulty. This modification does not change the method and only complicates data management and notation.

Denote a node's elevation by $u$ and define the pressure fraction, $z(h-u)$, by $z(h-u)=$ $\left(h-u-h_{m}\right) /\left(h_{s}-h_{m}\right)$. Suppose that $\gamma(t)$ is a bounded, smooth, monotonically increasing function which maps the interval $[0,1] \rightarrow[0,1]$. The POR at a node is defined by

$$
c(h)= \begin{cases}0 & \text { if } z(h-u) \leq 0 \\ d \gamma(z(h-u)) & \text { if } 0<z(h-u)<1 . \\ d & \text { if } z(h-u) \geq 1\end{cases}
$$

The inverse function of the POR, the head, $h_{i}(c)$ expressed as a function of outflow $c$ at node $i$, will be required for the development of the ASMFC which is the subject of this paper. But, the function $h_{i}(c)$ is not in general everywhere differentiable and so in its place a multivalued, sub-differential mapping is considered:

$$
h_{i}(c)= \begin{cases}\emptyset & \text { if } c<0 \\ \left(-\infty, h_{m}+u_{i}\right] & \text { if } c=0 \\ \left(h_{s}-h_{m}\right) \gamma^{-1}\left(\frac{c}{d_{i}}\right)+h_{m}+u_{i} & \text { if } 0<c<d_{i} . \\ {\left[h_{s}+u_{i},+\infty\right)} & \text { if } c=d_{i} \\ \emptyset & \text { if } c>d_{i}\end{cases}
$$

The definitions (1) and (2) are not, as they stand, applicable to the 2-side regularized Wagner or the logistic sigmoidal POR. The new method is, nevertheless, applicable for these PORs by adapting the intervals $\left([0,1]\right.$ and $\left.\left[0, d_{i}\right]\right)$ in the definitions of (1) and (2) (See Deuerlein et al. (2019) for details)

Note that, in what follows, we will carefully distinguish between the scalar variables, $h$, and the multivalued, sub-differential mapping, $h(c)$.

\section{THE PRESSURE DEPENDENT CONTENT MODEL AND THE ASM}


Denote the PDM content function

$$
C(\boldsymbol{q})=\sum_{j=1}^{n_{p}} \int_{0}^{q_{j}} \xi_{j}(s) d s-\boldsymbol{a}^{T} \boldsymbol{q}+\sum_{i=1}^{n_{j}} \int_{0}^{-\boldsymbol{e}_{i}^{T} \boldsymbol{A}^{T} \boldsymbol{q}} h_{i}(s) d s
$$

and define the set $U=\left\{\boldsymbol{q} \in \mathbb{R}^{n_{p}} \mid \boldsymbol{o} \leq-\boldsymbol{A}^{T} \boldsymbol{q} \leq \boldsymbol{d}\right\}$. For appropriate PORs the function $C(\boldsymbol{q})$ is strictly convex by virtue of the strict monotonicity of the head loss and consumption functions and it is also norm-coercive $(|C(\boldsymbol{q})| \rightarrow \infty$ if $\|\boldsymbol{q}\| \rightarrow \infty)$. The problem of finding $\min _{\boldsymbol{q} \in U} C(\boldsymbol{q})$ is associated with the Lagrangian $L(\boldsymbol{q}, \boldsymbol{h})=\sum_{j=1}^{n_{p}} \int_{0}^{q_{j}} \xi_{j}(s) d s-\boldsymbol{a}^{T} \boldsymbol{q}-\boldsymbol{h}^{T} \boldsymbol{A}^{T} \boldsymbol{q}-\sum_{i=1}^{n_{j}} \int_{h_{m}}^{h_{i}} c(s) d s$ and this leads to the unconstrained, equivalent problem of finding $\min _{q} \max _{h} L(\boldsymbol{q}, \boldsymbol{h})$ (see Elhay et al. (2016) for details). The gradient of $L(\boldsymbol{q}, \boldsymbol{h})$ is the familiar system

$$
\boldsymbol{f}(\boldsymbol{q}, \boldsymbol{h})=\left(\begin{array}{c}
\boldsymbol{G}(\boldsymbol{q}) \boldsymbol{q}-\boldsymbol{A} \boldsymbol{h}-\boldsymbol{a} \\
-\boldsymbol{A}^{T} \boldsymbol{q}-\boldsymbol{c}(\boldsymbol{h})
\end{array}\right) \stackrel{\text { def }}{=}\left(\begin{array}{c}
\boldsymbol{\rho}_{e} \\
\boldsymbol{\rho}_{m}
\end{array}\right)
$$

and the PDM steady-state heads and flows are found as the solution of $\boldsymbol{f}(\boldsymbol{q}, \boldsymbol{h})=\boldsymbol{o}$. Here $\boldsymbol{\rho}_{e}$ is the energy residual and $\boldsymbol{\rho}_{m}$ is the mass balance residual. The Jacobian of $\boldsymbol{f}$ is

$$
\nabla_{\boldsymbol{q}, \boldsymbol{h}} \boldsymbol{f}(\boldsymbol{q}, \boldsymbol{h})=\left(\begin{array}{cc}
\boldsymbol{F}(\boldsymbol{q}) & -\boldsymbol{A} \\
-\boldsymbol{A}^{T} & -\boldsymbol{E}(\boldsymbol{h})
\end{array}\right)
$$

where $\boldsymbol{F}(\boldsymbol{q})$ and $\boldsymbol{E}(\boldsymbol{h})$ are diagonal matrices which are such that (i) the terms on the diagonal of $\boldsymbol{F}(\boldsymbol{q})$ are the $q$-derivatives of the corresponding terms in $\boldsymbol{G}(\boldsymbol{q}) \boldsymbol{q}$ and (ii) the terms on the diagonal of $\boldsymbol{E}$ are the $h$-derivatives of the corresponding terms in $\boldsymbol{c}(\boldsymbol{h})$.

From this formulation the authors developed an ASM for the equivalent box-constrained minimization problem in which the nodal outflows, $\boldsymbol{c}$ are considered additional unknowns. The restriction of the content function $C(\boldsymbol{q}, \boldsymbol{c})$, where the components of $\boldsymbol{c}$ are defined only on the intervals $0 \leq c_{i} \leq d_{i}$,

$$
C(\boldsymbol{q}, \boldsymbol{c})=\sum_{j=1}^{n_{p}} \int_{0}^{q_{j}} \xi_{j}(s) d s-\boldsymbol{a}^{T} \boldsymbol{q}+\boldsymbol{c}^{T}\left(\boldsymbol{u}+h_{m}\right)+\sum_{\substack{1 \leq i \leq n_{j} \\ d_{i}>0}}\left(h_{s}-h_{m}\right) \int_{0}^{c_{i}} \gamma^{-1}\left(\frac{s}{d_{i}}\right) d s
$$

is minimized subject to $-\boldsymbol{A}^{T} \boldsymbol{q}-\boldsymbol{c}=\boldsymbol{o},-\boldsymbol{c} \leq \boldsymbol{o}$ and $\boldsymbol{c} \leq \boldsymbol{d}$. Let $\boldsymbol{\psi}(\boldsymbol{c})$ denote (for later use) a vector whose components are the individual terms under the summation sign in the last term in Eq. (6). In order for a solution to exist it is only necessary that the polyhedral constraint set $-\boldsymbol{A}^{T} \boldsymbol{q}-\boldsymbol{c}=\boldsymbol{o}$ and $\boldsymbol{o} \leq \boldsymbol{c} \leq \boldsymbol{d}$ be non-empty. The link flows $\boldsymbol{q}=\boldsymbol{o}$ and nodal outflows $\boldsymbol{c}=\boldsymbol{o}$ are trivially feasible solutions for the constraint set and so the PDM problem consists of the minimization of a strictly convex content function formulated in terms of unknown flows 
$\boldsymbol{q}, \boldsymbol{c}$ over a polyhedral set. Associated with the problem

$$
\min _{\boldsymbol{q}, \boldsymbol{c}} C(\boldsymbol{q}, \boldsymbol{c}), \text { subject to }-\boldsymbol{A}^{T} \boldsymbol{q}-\boldsymbol{c}=\boldsymbol{o}, \quad \boldsymbol{o} \leq \boldsymbol{c} \leq \boldsymbol{d}
$$

is the Lagrangian

$$
\begin{gathered}
L(\boldsymbol{q}, \boldsymbol{c}, \boldsymbol{h}, \overline{\boldsymbol{\lambda}}, \overline{\boldsymbol{\mu}})=\sum_{j=1}^{n_{p}} \int_{0}^{q_{j}} \xi_{j}(s) d s-\boldsymbol{a}^{T} \boldsymbol{q}+\mathbf{1}^{T} \boldsymbol{\psi}(\boldsymbol{c})+\boldsymbol{c}^{T}\left(\boldsymbol{u}+h_{m}\right)-\boldsymbol{h}^{T}\left(\boldsymbol{A}^{T} \boldsymbol{q}+\boldsymbol{c}\right) \\
+\overline{\boldsymbol{\mu}}^{T}(\boldsymbol{c}-\boldsymbol{d})-\overline{\boldsymbol{\lambda}}^{T} \boldsymbol{c}, \quad \overline{\boldsymbol{\lambda}} \geq \boldsymbol{o}, \quad \overline{\boldsymbol{\mu}} \geq \boldsymbol{o}
\end{gathered}
$$

where $\mathbf{1}=(1,1, \ldots, 1)^{T}$, the heads, $\boldsymbol{h}$, represent the non-negative Lagrange multipliers for the mass balance equality constraint and $\overline{\boldsymbol{\lambda}}$ and $\overline{\boldsymbol{\mu}}$ are the non-negative Lagrange multipliers for the inequality constraints on the outflows, $\boldsymbol{c}$.

\section{THE CONTENT MODEL FOR PRESSURE DRIVEN DEMANDS WITH LINK FLOW CONSTRAINTS}

If flow constraints are added to the system, the minimization problem (7) becomes

$$
\min _{q, c} C(\boldsymbol{q}, \boldsymbol{c}) \text { subject to },-\boldsymbol{A}^{T} \boldsymbol{q}-\boldsymbol{c}=\boldsymbol{o},-\boldsymbol{c} \leq \boldsymbol{o}, \boldsymbol{c} \leq \boldsymbol{d}, \boldsymbol{q}-\boldsymbol{q}_{\max } \leq 0,-\boldsymbol{q}+\boldsymbol{q}_{\min } \leq 0 .
$$

As a formalism, any links which do not have finite constraints are considered to lie in the interval $(-\infty, \infty)$.

The notation we adopt for Lagrange multipliers is as follows. The symbols $\overline{\boldsymbol{\kappa}}, \overline{\boldsymbol{\nu}} \in \mathbb{R}^{n_{p}}$ and denote generic Lagrange multiplier variables but $\widehat{\boldsymbol{\kappa}}, \widehat{\boldsymbol{\nu}}$ (e.g. in (11)) have generally smaller dimension: $\widehat{\boldsymbol{\kappa}}$ has the dimension of the number of constraints active at the lower link flow constraint boundary and $\widehat{\boldsymbol{\nu}}$ has the dimension of the number of constraints active at the upper link flow constraint boundary, in both cases, at the solution point. The Lagrange multipliers $\boldsymbol{\kappa}^{(m)}, \boldsymbol{\nu}^{(m)}$ (e.g. in (19)) also have generally smaller dimensions but these can change from one iteration to the next: their dimensions match the number of (lower and upper, respectively) link flow constraints which are active at the $m$-th iteration.

The associated Lagrangian is now

$$
\begin{gathered}
L(\boldsymbol{q}, \boldsymbol{c}, \boldsymbol{h}, \overline{\boldsymbol{\lambda}}, \overline{\boldsymbol{\mu}}, \overline{\boldsymbol{\kappa}}, \overline{\boldsymbol{\nu}})=\sum_{j=1}^{n_{p}} \int_{0}^{q_{j}} \xi_{j}(s) d s-\boldsymbol{a}^{T} \boldsymbol{q}+\mathbf{1}^{T} \boldsymbol{\psi}(\boldsymbol{c})+\boldsymbol{c}^{T}\left(\boldsymbol{u}+h_{m}\right)-\boldsymbol{h}^{T}\left(\boldsymbol{A}^{T} \boldsymbol{q}+\boldsymbol{c}\right) \\
+\overline{\boldsymbol{\mu}}^{T}(\boldsymbol{c}-\boldsymbol{d})-\overline{\boldsymbol{\lambda}}^{T} \boldsymbol{c}+\overline{\boldsymbol{\kappa}}^{T}\left(-\boldsymbol{q}+\boldsymbol{q}_{\min }\right)+\overline{\boldsymbol{\nu}}^{T}\left(\boldsymbol{q}-\boldsymbol{q}_{\max }\right) \\
\overline{\boldsymbol{\lambda}} \geq \boldsymbol{o}, \quad \overline{\boldsymbol{\mu}} \geq \boldsymbol{o}, \quad \overline{\boldsymbol{\kappa}} \geq \boldsymbol{o}, \quad \overline{\boldsymbol{\nu}} \geq \boldsymbol{o}
\end{gathered}
$$

with $\overline{\boldsymbol{\lambda}}$ and $\overline{\boldsymbol{\mu}}$ the Lagrange multipliers for the outflow constraints and $\overline{\boldsymbol{\kappa}}$ and $\overline{\boldsymbol{\nu}}$, the Lagrange multipliers for the link flow constraints. The necessary and sufficient KKT conditions for a 
minimum are, considering the complementary slackness conditions for the inequality constraints (and having zero Lagrange multipliers for the inactive constraints),

$$
\begin{aligned}
\boldsymbol{\xi}(\boldsymbol{q})-\boldsymbol{A} \boldsymbol{h}-\boldsymbol{a}-\boldsymbol{L}_{q}^{T} \widehat{\boldsymbol{\kappa}}+\boldsymbol{U}_{q}^{T} \widehat{\boldsymbol{\nu}} & =\boldsymbol{o} \\
\boldsymbol{h}(\boldsymbol{c})-\boldsymbol{h}-\boldsymbol{L}_{c}^{T} \widehat{\boldsymbol{\lambda}}+\boldsymbol{U}_{c}^{T} \widehat{\boldsymbol{\mu}} & =\boldsymbol{o} \\
-\boldsymbol{A}^{T} \boldsymbol{q}-\boldsymbol{c} & =\boldsymbol{o} \\
-\boldsymbol{L}_{c} \boldsymbol{c} & =\boldsymbol{o} \\
\boldsymbol{U}_{c}(\boldsymbol{c}-\boldsymbol{d}) & =\boldsymbol{o} \\
\boldsymbol{L}_{q}\left(-\boldsymbol{q}+\boldsymbol{q}_{\min }\right) & =\boldsymbol{o} \\
\boldsymbol{U}_{q}\left(\boldsymbol{q}-\boldsymbol{q}_{\max }\right) & =\boldsymbol{o} \\
\widehat{\boldsymbol{\lambda}} \geq \boldsymbol{o}, \widehat{\boldsymbol{\mu}} \geq \boldsymbol{o}, \widehat{\boldsymbol{\kappa}} \geq \boldsymbol{o}, \widehat{\boldsymbol{\nu}} & \geq \boldsymbol{o}
\end{aligned}
$$

where (i) $\widehat{\boldsymbol{\lambda}}, \widehat{\boldsymbol{\mu}}, \widehat{\boldsymbol{\kappa}}$ and $\widehat{\boldsymbol{\nu}}$ are the Lagrange multipliers of the active PORs and link flow bounds at the solution, (ii) $\boldsymbol{L}_{c}$ and $\boldsymbol{U}_{c}$ are matrices made up of the rows of an identity matrix which correspond to the active set indices for the lower and upper constraints for the outflows and (iii) $\boldsymbol{L}_{q}$ and $\boldsymbol{U}_{q}$ are the matrices made up of the rows of identity matrices which correspond to the active set indices for the lower and upper link flow constraints. Eqs. (14) - (17) represent the binding constraints and Eqs. (13)-(17) define what is known as the linear independence constraint qualification (LICQ): the matrix of the gradient of the active inequality constraints together with the gradient of the equality constraints. If this matrix has full rank then LICQ is said to hold. Now, if the head losses and the PORs are strictly monotonic then the content function is strictly convex and there is at most one solution. An LP can be used to determine existence. Thus, the KKT conditions together with norm-coercivity of the content function, convexity and the LICQ imply uniqueness of both heads and flows. By contrast, if the LICQ is violated then the heads may not be unique because the Lagrange multipliers are not uniquely defined. However, the flows are always unique by virtue of strict convexity.

In computation, convergence failures for this problem are often a result of the violation of the LICQ. Even if the LP establishes that the LICQ holds at the solution point, the LICQ may be violated at an intermediate iteration on the way to the solution. This is manifest in the singularity of the Schur complement (Golub \& Van Loan 1983, 58), a matrix which is key to the solution process. 
Denote $\boldsymbol{M}=\operatorname{diag}\left\{h_{1}^{\prime}(c), h_{2}^{\prime}(c), \ldots, h_{n_{j}}^{\prime}(c)\right\}$. The Newton method for (11) to (17) is

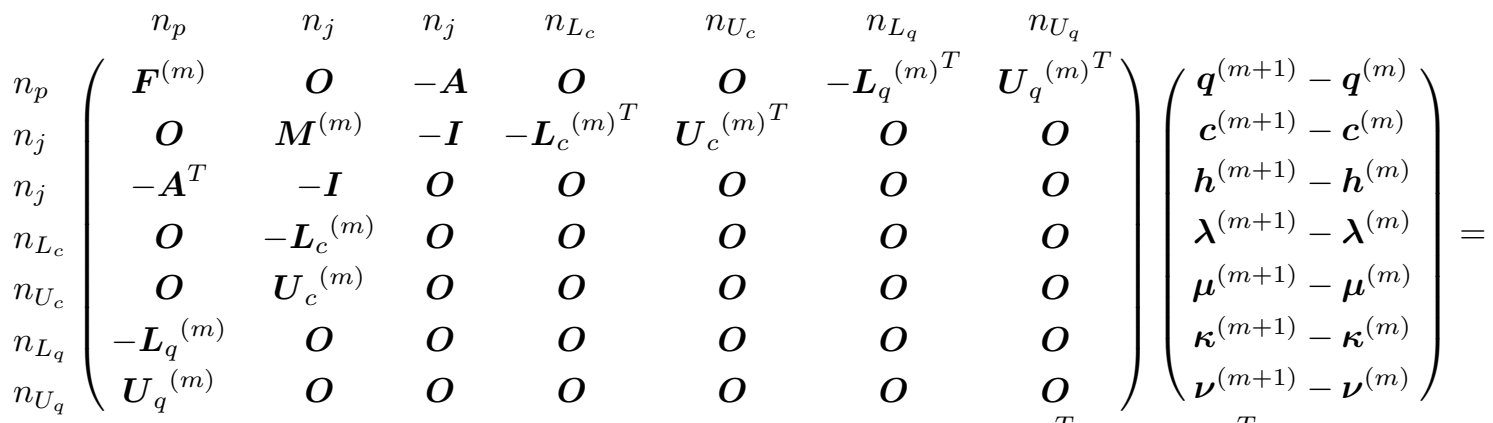

$$
\begin{aligned}
& -\left(\begin{array}{c}
\boldsymbol{G}^{(m)} \boldsymbol{q}^{(m)}-\boldsymbol{A} \boldsymbol{h}^{(m)}-\boldsymbol{a}-\boldsymbol{L}_{q}{ }^{(m)^{T}} \boldsymbol{\kappa}+\boldsymbol{U}_{q}{ }^{(m)^{T}} \boldsymbol{\nu} \\
\boldsymbol{h}\left(\boldsymbol{c}^{(m)}\right)-\boldsymbol{h}^{(m)}-\boldsymbol{L}_{c}{ }^{(m)^{T}} \boldsymbol{\lambda}^{(m)}+\boldsymbol{U}_{c}^{(m)^{T}} \boldsymbol{\mu}^{(m)} \\
-\boldsymbol{A}^{T} \boldsymbol{q}^{(m)}-\boldsymbol{c}^{(m)} \\
-\boldsymbol{L}_{c}^{(m)} \boldsymbol{c}^{(m)} \\
\boldsymbol{U}_{c}^{(m)}\left(\boldsymbol{c}^{(m)}-\boldsymbol{d}\right) \\
\boldsymbol{L}_{q}^{(m)}\left(-\boldsymbol{q}^{(m)}+\boldsymbol{q}_{\min }\right) \\
\boldsymbol{U}_{q}^{(m)}\left(\boldsymbol{q}^{(m)}-\boldsymbol{q}_{\max }\right)
\end{array}\right)
\end{aligned}
$$

The rows and columns of matrices $\boldsymbol{M}^{(m)}, \boldsymbol{I}$ and $\boldsymbol{A}$ are now partitioned into a block threeby-three configuration. The matrix $\boldsymbol{M}^{(m)}$ has three diagonal blocks: the block $\boldsymbol{M}_{c_{b}}^{(m)}$ which represents those nodes at which the head lies between the minimum and service pressure head, the block $\boldsymbol{M}_{c_{l}}^{(m)}$ which represents the active sets of those nodes at which no outflow is possible because the pressure is below the minimum pressure head and the block $\boldsymbol{M}_{c_{u}}^{(m)}$ which represents the active sets of those nodes at which the outflow is at the nominal demand, $d$, because the head is at least at the service pressure head level:

$$
\boldsymbol{M}^{(m)}=\begin{array}{ccc}
n_{c_{b}} & n_{c_{l}} & n_{c_{u}} \\
& n_{c_{b}} \\
n_{c_{l}} \\
n_{c_{u}}
\end{array}\left(\begin{array}{lll}
\boldsymbol{M}_{c_{b}}^{(m)} & & \\
& \boldsymbol{M}_{c_{l}}^{(m)} & \\
& & \boldsymbol{M}_{c_{u}}^{(m)}
\end{array}\right)
$$

The ANIM, $\boldsymbol{A}$ is similarly partitioned into block three-by-three form where the block's first subscript refers to rows (links) sets and the second subscript refers to columns (nodes) sets: the subscripts indicate (i) the set for which the constraints are not active, $b$, (ii) the set for which the lower constraint is active, $l$, and (iii) the set for which the upper constraint is active, $u$.

$$
\begin{aligned}
& n_{c_{b}} \quad n_{c_{l}} \quad n_{c_{u}} \\
& \boldsymbol{A}=\underset{n_{q_{l}}}{n_{q_{u}}}\left(\begin{array}{lll}
n_{q_{b}} \\
\boldsymbol{A}_{b b} & \boldsymbol{A}_{b l} & \boldsymbol{A}_{b u} \\
\boldsymbol{A}_{l b} & \boldsymbol{A}_{l l} & \boldsymbol{A}_{l u} \\
\boldsymbol{A}_{u b} & \boldsymbol{A}_{u l} & \boldsymbol{A}_{u u}
\end{array}\right)=\left(\begin{array}{c}
\boldsymbol{A}_{b .} \\
\boldsymbol{A}_{l .} \\
\boldsymbol{A}_{u .}
\end{array}\right)
\end{aligned}
$$


Thus, $\boldsymbol{A}_{b l} \in \mathbb{R}^{n_{q_{b}} \times n_{c_{l}}}$ represents those links for which the link flows are between the upper and lower constraint boundaries and the nodes for which the pressure heads are below the minimum pressure head. The various identity matrix partition blocks are designated by $\boldsymbol{I}_{q}$. for link flows and $\boldsymbol{I}_{c .}$ for outflows. If the matrix $\boldsymbol{G}^{(m)}$, and the head loss derivatives matrix $\boldsymbol{F}^{(m)}$ and the identity are partitioned conformally with $\boldsymbol{M}^{(m)}$ and $\boldsymbol{A}$ the resulting system is

$$
\boldsymbol{P}^{(m)}\left(\boldsymbol{x}^{(m+1)}-\boldsymbol{x}^{(m)}\right)=\boldsymbol{r}^{(m)}
$$

where, dropping subscripts and superscripts where there is no ambiguity,

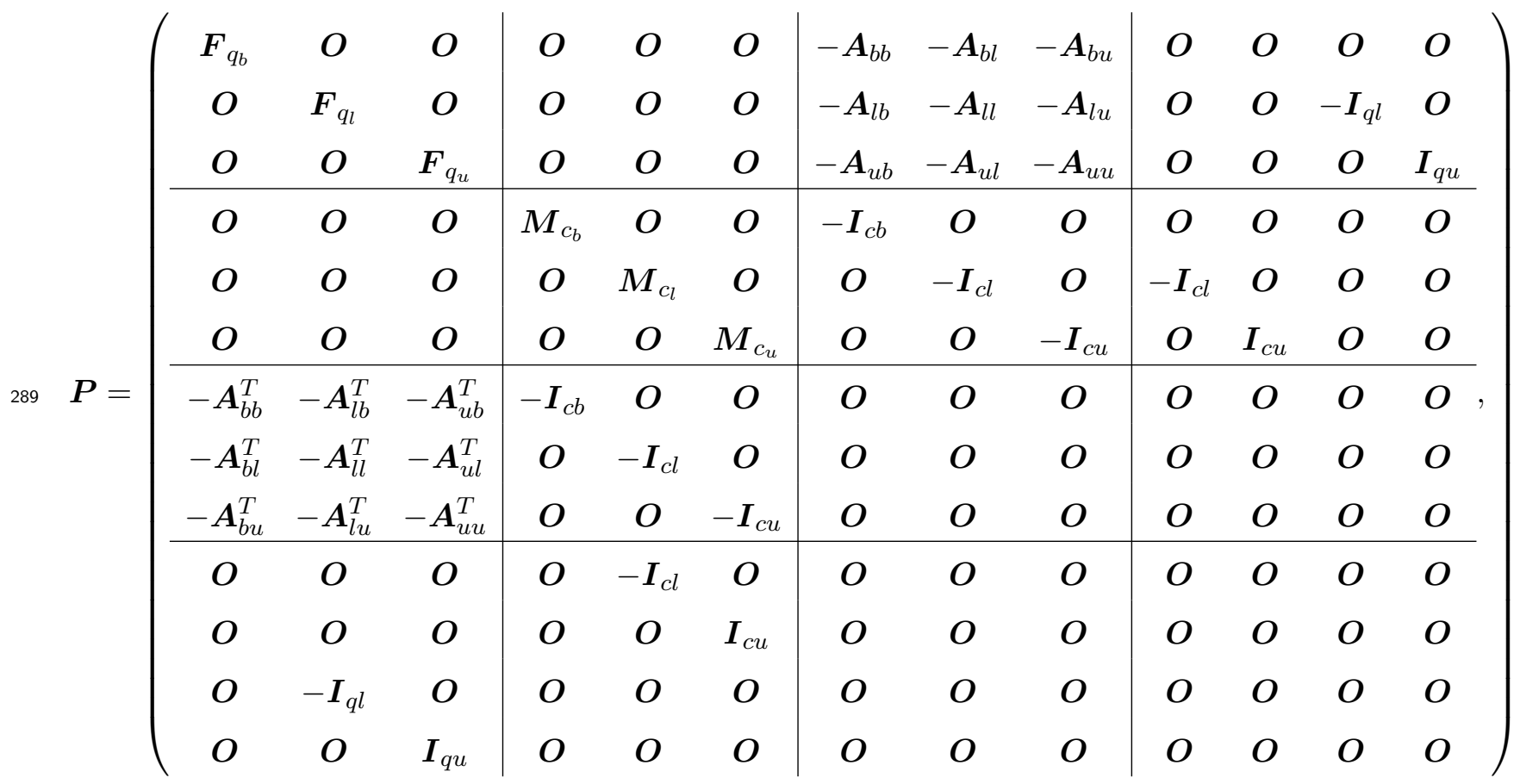




$$
\boldsymbol{x}^{(m+1)}-\boldsymbol{x}^{(m)}=\left(\begin{array}{c}
\boldsymbol{q}_{b}^{(m+1)}-\boldsymbol{q}_{b}^{(m)} \\
\boldsymbol{q}_{l}^{(m+1)}-\boldsymbol{q}_{l}^{(m)} \\
\frac{\boldsymbol{q}_{u}^{(m+1)}-\boldsymbol{q}_{u}^{(m)}}{\boldsymbol{c}_{b}^{(m+1)}-\boldsymbol{c}_{b}^{(m)}} \\
\boldsymbol{c}_{l}^{(m+1)}-\boldsymbol{c}_{l}^{(m)} \\
\boldsymbol{c}_{u}^{(m+1)}-\boldsymbol{c}_{u}^{(m)} \\
\boldsymbol{h}_{c_{b}}^{(m+1)}-\boldsymbol{h}_{c_{b}}^{(m)} \\
\boldsymbol{h}_{c_{l}}^{(m+1)}-\boldsymbol{h}_{c_{l}}^{(m)} \\
\boldsymbol{h}_{c_{u}}^{(m+1)}-\boldsymbol{h}_{c_{u}}^{(m)} \\
\boldsymbol{\lambda}^{(m+1)}-\boldsymbol{\lambda}^{(m)} \\
\boldsymbol{\mu}^{(m+1)}-\boldsymbol{\mu}^{(m)} \\
\boldsymbol{\kappa}^{(m+1)}-\boldsymbol{\kappa}^{(m)} \\
\boldsymbol{\nu}^{(m+1)}-\boldsymbol{\nu}^{(m)}
\end{array}\right)
$$

292

$$
\boldsymbol{r}^{(m)}=-\left(\begin{array}{c}
\boldsymbol{G}_{q_{b}} \boldsymbol{q}_{b}^{(m)}-\boldsymbol{A}_{b b} \boldsymbol{h}_{c_{b}}^{(m)}-\boldsymbol{A}_{b l} \boldsymbol{h}_{c_{l}}^{(m)}-\boldsymbol{A}_{b u} \boldsymbol{h}_{c_{u}}^{(m)}-\boldsymbol{a}_{q_{b}} \\
\boldsymbol{G}_{q_{l}} \boldsymbol{q}_{l}^{(m)}-\boldsymbol{A}_{l b} \boldsymbol{h}_{c_{b}}^{(m)}-\boldsymbol{A}_{l l} \boldsymbol{h}_{c_{l}}^{(m)}-\boldsymbol{A}_{l u} \boldsymbol{h}_{c_{u}}^{(m)}-\boldsymbol{a}_{q_{l}}-\boldsymbol{\kappa}^{(m)} \\
\boldsymbol{G}_{q_{u}} \boldsymbol{q}_{u}^{(m)}-\boldsymbol{A}_{u b} \boldsymbol{h}_{c_{b}}^{(m)}-\boldsymbol{A}_{u l} \boldsymbol{h}_{c_{l}}^{(m)}-\boldsymbol{A}_{u u} \boldsymbol{h}_{c_{u}}^{(m)}-\boldsymbol{a}_{q_{u}}+\boldsymbol{\nu}^{(m)} \\
\boldsymbol{h}\left(\boldsymbol{c}_{b}^{(m)}\right)-\boldsymbol{h}_{c_{b}}^{(m)} \\
-\boldsymbol{h}_{c_{l}}^{(m)}+h_{m}+\boldsymbol{u}_{c_{l}}-\boldsymbol{\lambda}^{(m)} \\
-\boldsymbol{h}_{c_{u}}^{(m)}+h_{s}+\boldsymbol{u}_{c_{u}}+\boldsymbol{\mu}^{(m)} \\
-\boldsymbol{A}_{b b}^{T} \boldsymbol{q}_{b}^{(m)}-\boldsymbol{A}_{l b}^{T} \boldsymbol{q}_{l}^{(m)}-\boldsymbol{A}_{u b}^{T} \boldsymbol{q}_{u}^{(m)}-\boldsymbol{c}_{b}^{(m)} \\
-\boldsymbol{A}_{b l}^{T} \boldsymbol{q}_{b}^{(m)}-\boldsymbol{A}_{l l}^{T} \boldsymbol{q}_{l}^{(m)}-\boldsymbol{A}_{u l}^{T} \boldsymbol{q}_{u}^{(m)}-\boldsymbol{c}_{l}^{(m)} \\
-\boldsymbol{A}_{b u}^{T} \boldsymbol{q}_{b}^{(m)}-\boldsymbol{A}_{l u}^{T} \boldsymbol{q}_{l}^{(m)}-\boldsymbol{A}_{u u}^{T} \boldsymbol{q}_{u}^{(m)}-\boldsymbol{c}_{u}^{(m)} \\
-\boldsymbol{c}_{l}^{(m)} \\
\boldsymbol{c}_{u}^{(m)}-\boldsymbol{d}_{u} \\
-\boldsymbol{q}_{l}^{(m)}+\boldsymbol{q}_{l, m i n} \\
\boldsymbol{q}_{u}^{(m)}-\boldsymbol{q}_{u, m a x}
\end{array}\right)
$$

In this formulation of the problem the heads are unconstrained but for convenience we denote by $\boldsymbol{h}_{c_{b}}$ the heads which are between the minimum and service pressure heads, by $\boldsymbol{h}_{c_{l}}$ the heads which are at or below the minimum pressure head and by $\boldsymbol{h}_{c_{u}}$ the heads which are at or above the service pressure head. By analytically solving for some of the unknowns in terms of the others (see the Appendix for details and the notation defining $\widehat{\boldsymbol{I}}_{c b}$ and $\widehat{\boldsymbol{I}}_{c u}$ ) the system 
(20) can be rewritten

$$
\left(\begin{array}{cc|c}
\boldsymbol{F}_{q_{b}} & \boldsymbol{O} & -\boldsymbol{A}_{b .} \\
\boldsymbol{O} & \boldsymbol{M}_{c_{b}} & -\widehat{\boldsymbol{I}}_{c b} \\
\hline-\boldsymbol{A}_{b .}^{T} & -\widehat{\boldsymbol{I}}_{c b}^{T} & \boldsymbol{O}
\end{array}\right)\left(\begin{array}{c}
\boldsymbol{q}_{b}^{(m+1)}-\boldsymbol{q}_{b}^{(m)} \\
\frac{\boldsymbol{c}_{b}^{(m+1)}-\boldsymbol{c}_{b}^{(m)}}{\boldsymbol{h}^{(m+1)}-\boldsymbol{h}^{(m)}}
\end{array}\right)=-\left(\begin{array}{c}
\boldsymbol{G}_{q_{b}} \boldsymbol{q}_{b}^{(m)}-\boldsymbol{A}_{b .} \boldsymbol{h}^{(m)}-\boldsymbol{a}_{q_{b}} \\
\boldsymbol{h}\left(\boldsymbol{c}_{b}^{(m)}\right)-\boldsymbol{h}_{c_{b}}^{(m)} \\
\hline-\boldsymbol{A}_{b .}^{T} \boldsymbol{q}_{b}^{(m)}-\boldsymbol{A}_{l .}^{T} \boldsymbol{q}_{l, m i n}-\boldsymbol{A}_{u, .}^{T} \boldsymbol{q}_{u, m a x}-\widehat{\boldsymbol{I}}_{c b}^{T} \boldsymbol{c}_{b}^{(m)}-\widehat{\boldsymbol{I}}_{c u}^{T} \boldsymbol{d}_{u}
\end{array}\right)
$$

Denote the Schur complement of the matrix on the left of (21) by

$$
\boldsymbol{V}_{b}^{(m)}=\left(\boldsymbol{A}_{b .}^{T} \boldsymbol{F}_{q_{b}}^{-1} \boldsymbol{A}_{b .}+\widehat{\boldsymbol{I}}_{c b}^{T} \boldsymbol{M}_{c b}^{-1} \widehat{\boldsymbol{I}}_{c b}\right) \in \mathbb{R}^{n_{j} \times n_{j}}
$$

The system (21) leads to three update equations

$$
\begin{gathered}
\boldsymbol{V}_{b}^{(m)}\left(\boldsymbol{h}^{(m+1)}-\boldsymbol{h}^{(m)}\right)=-\boldsymbol{A}^{T} \boldsymbol{q}^{(m)}-\widehat{\boldsymbol{I}}_{c b}^{T} \boldsymbol{c}_{b}^{(m)}-\widehat{\boldsymbol{I}}_{c u}^{T} \boldsymbol{d}_{u} \\
+\boldsymbol{A}_{b .}^{T} \boldsymbol{F}_{q_{b}}^{-1}\left(\boldsymbol{G}_{q_{b}} \boldsymbol{q}_{b}^{(m)}-\boldsymbol{A}_{b .} \boldsymbol{h}^{(m)}-\boldsymbol{a}_{q_{b}}\right)+\widehat{\boldsymbol{I}}_{c b}^{T} \boldsymbol{M}_{c_{b}}^{-1}\left(\boldsymbol{h}\left(\boldsymbol{c}_{b}^{(m)}\right)-\boldsymbol{h}_{c_{b}}^{(m)}\right) \\
\boldsymbol{q}_{b}^{(m+1)}=\boldsymbol{q}_{b}^{(m)}-\boldsymbol{F}_{q_{b}}^{-1}\left(\boldsymbol{G}_{q_{b}} \boldsymbol{q}_{b}^{(m)}-\boldsymbol{A}_{b .} \boldsymbol{h}^{(m+1)}-\boldsymbol{a}_{q_{b}}\right) \\
\boldsymbol{c}_{b}^{(m+1)}=\boldsymbol{c}_{b}^{(m)}-\boldsymbol{M}_{c_{b}}^{-1}\left(\boldsymbol{h}\left(\boldsymbol{c}_{b}^{(m)}\right)-\boldsymbol{h}_{c_{b}}^{(m+1)}\right) .
\end{gathered}
$$

The updated Lagrange multipliers are given by

$$
\begin{gathered}
\boldsymbol{\lambda}^{(m+1)}=h_{m}+\boldsymbol{u}_{c_{l}}-\boldsymbol{h}_{c_{l}}^{(m+1)}, \quad \boldsymbol{\mu}^{(m+1)}=\boldsymbol{h}_{c_{u}}^{(m+1)}-\boldsymbol{u}_{c_{u}}-h_{s}, \\
\boldsymbol{\kappa}^{(m+1)}=\boldsymbol{G}_{q_{l}} \boldsymbol{q}_{l}^{(m)}-\boldsymbol{A}_{l b} \boldsymbol{h}_{c_{b}}^{(m+1)}-\boldsymbol{A}_{l l} \boldsymbol{h}_{c_{l}}^{(m+1)}-\boldsymbol{A}_{l u} \boldsymbol{h}_{c_{u}}^{(m+1)}-\boldsymbol{a}_{q_{l}} \\
\boldsymbol{\nu}^{(m+1)}=-\left(\boldsymbol{G}_{q_{u}} \boldsymbol{q}_{u}^{(m)}-\boldsymbol{A}_{u b} \boldsymbol{h}_{c_{b}}^{(m+1)}-\boldsymbol{A}_{u l} \boldsymbol{h}_{c_{l}}^{(m+1)}-\boldsymbol{A}_{u u} \boldsymbol{h}_{c_{u}}^{(m+1)}-\boldsymbol{a}_{q_{u}}\right)
\end{gathered}
$$

Eqs. (23)-(28), all but the first of which are explicit, form the basis of an active set method to solve this constrained optimization problem. Before presenting the algorithm for the new method a few preliminaries are necessary.

\section{The index sets}

Let $N_{q}$ be the index set of links with flow constraints and $N_{c}$ be the set of all nodes with unknown-head and $d_{i}>0$. Six index sets are defined: three, $\mathcal{I}_{q_{b}}, \mathcal{I}_{q_{l}}$, and $\mathcal{I}_{q_{u}}$, for the links which are flow constrained and three, $\mathcal{I}_{c_{b}}, \mathcal{I}_{c_{l}}$, and $\mathcal{I}_{c_{u}}$, which are defined only for the nodes at which the nominal demand is positive, $d_{i}>0$. All the links which have no finite flow constraints are considered to satisfy $-\infty<q_{i}^{(m+1)}<\infty$ and so always fall into the interior of their constraint intervals: their constraints are never active. Note that the elements and the number of elements 
in these sets can change from one iteration to the next. Strictly speaking, the sets represent the states of the link flows and the outflows only at the end of each iteration (i.e. after any flows outside the constraint intervals have been projected onto the constraint boundaries).

The first three sets in question are the link flow index sets. Set $\mathcal{I}_{q_{b}}$ has the indices of the links for which the flows are either in the interior of the constraint interval or are unconstrained. The other two sets, $\mathcal{I}_{q_{l}}$ and $\mathcal{I}_{q_{u}}$, hold the indices of the links for which the constraints are active. They can, in fact, be considered together as one index set for all the inequality constraints but they are treated separately here in the interests of clarity.

The last three sets are the outflow index sets. The set $\mathcal{I}_{c_{b}}$ holds the indices of the nodes at which the head is between the minimum head, $h_{m}$, and service head $h_{s}$, and the other two sets hold the indices of the nodes at which the head is either less than or equal to $h_{m}$ or greater than or equal to $h_{s}$. The sets $\mathcal{I}_{c_{u}}$ and $\mathcal{I}_{c_{l}}$ can also be considered together. The sets themselves are defined by:

(a)

$$
\mathcal{I}_{q_{b}}^{(m+1)}=\left\{j \in N_{q} \mid\left(q_{j}^{(m+1)}>q_{m i n, j} \wedge q_{j}^{(m+1)}<q_{\max , j}\right) \vee\right.
$$

$$
\left.\left(q_{j}^{(m+1)}=q_{m i n, j} \wedge \kappa_{j}^{(m+1)}<0\right) \vee\left(q_{j}^{(m+1)}=q_{\max , j} \wedge \nu_{j}^{(m+1)}<0\right)\right\}
$$

(b) $\mathcal{I}_{q_{l}}^{(m+1)}=\left\{j \in N_{q} \mid\left(q_{j}^{(m+1)}<q_{m i n, j}\right) \vee\left(q_{j}^{(m+1)}=q_{m i n, j} \wedge \kappa_{j}^{(m+1)} \geq 0\right)\right\}$

(c) $\mathcal{I}_{q_{u}}^{(m+1)}=\left\{j \in N_{q} \mid\left(q_{j}^{(m+1)}>q_{\max , j}\right) \vee\left(q_{j}^{(m+1)}=q_{\max , j} \wedge \nu_{j}^{(m+1)} \geq 0\right)\right\}$. and

$$
\begin{aligned}
\mathcal{I}_{c_{b}}^{(m+1)}=\left\{i \in N_{c} \mid d_{i}>0\right. & \wedge\left(\left(c_{i}^{(m+1)}>0 \wedge c_{i}^{(m+1)}<d_{i}\right) \vee\right. \\
\left(c_{i}^{(m+1)}\right. & \left.\left.\left.=0 \wedge \lambda_{i}^{(m+1)}<0\right) \vee\left(c_{i}^{(m+1)}=d_{i} \wedge \mu_{i}^{(m+1)}<0\right)\right)\right\}
\end{aligned}
$$

(e) $\mathcal{I}_{c_{l}}^{(m+1)}=\left\{i \in N_{c} \mid d_{i}>0 \wedge\left(c_{i}^{(m+1)}<0 \vee\left(c_{i}^{(m+1)}=0 \wedge \lambda_{i}^{(m+1)} \geq 0\right)\right)\right\}$

(f) $\mathcal{I}_{c_{u}}^{(m+1)}=\left\{i \in N_{c} \mid d_{i}>0 \wedge\left(c_{i}^{(m+1)}>d_{i} \vee\left(c_{i}^{(m+1)}=d_{i} \wedge \mu_{i}^{(m+1)} \geq 0\right)\right)\right\}$.

\section{Set assignment algorithms}

Once the heads, link flows, outflows and Lagrange multipliers have all been updated, the links need to be classified according to their values and the values of their associated Lagrange multipliers. 
Thus, for each link flow $q_{j}$

(a) If $q_{\text {min }, j}<q_{j}<q_{\max , j}$ then $j \rightarrow \mathcal{I}_{q_{b}}$

(b) If $q_{j}<q_{\text {min, } j}$ then $j \rightarrow \mathcal{I}_{q_{l}}$

(c) If $q_{j}>q_{\max , j}$ then $j \rightarrow \mathcal{I}_{q_{u}}$

(d) If $q_{j}=q_{m i n, j}$ then

(i) If $\kappa_{j} \geq 0$ then $j \rightarrow \mathcal{I}_{q_{l}}$

(ii) otherwise $j \rightarrow \mathcal{I}_{q_{b}}$.

(e) If $q_{j}=q_{\max , j}$ then

(i) If $\nu_{j} \geq 0$ then $j \rightarrow \mathcal{I}_{q_{u}}$

(ii) otherwise $j \rightarrow \mathcal{I}_{q_{b}}$.

Similarly, the outflows are assigned to their index sets by the following algorithm. For each node with $d_{i}>0$

(a) If $0<c_{i}<d_{i}$ then $i \rightarrow \mathcal{I}_{c_{b}}$

(b) If $c_{i}<0$ then $i \rightarrow \mathcal{I}_{c_{l}}$

(c) If $c_{i}>d_{i}$ then $i \rightarrow \mathcal{I}_{c_{u}}$

(d) If $c_{i}=0$ then

(i) If $\lambda_{i} \geq 0$ then $i \rightarrow \mathcal{I}_{c_{l}}$

(ii) otherwise $i \rightarrow \mathcal{I}_{c_{b}}$.

(e) If $c_{i}=d_{i}$ then

(i) If $\mu_{i} \geq 0$ then $i \rightarrow \mathcal{I}_{c_{u}}$

(ii) otherwise $i \rightarrow \mathcal{I}_{c_{b}}$.

\section{Initialization}

Initial heads $\boldsymbol{h}^{(0)}$, link flows $\boldsymbol{q}^{(0)}$, nodal outflows $\boldsymbol{c}^{(0)}$, Lagrange multipliers $\boldsymbol{\lambda}^{(0)}, \boldsymbol{\mu}^{(0)}, \boldsymbol{\kappa}^{(0)}$, $\boldsymbol{\mu}^{(0)}$, and the corresponding index sets $\mathcal{I}_{q_{b}}, \mathcal{I}_{q_{l}}, \mathcal{I}_{q_{u}}, \mathcal{I}_{c_{b}}, \mathcal{I}_{c_{l}}, \mathcal{I}_{c_{u}}$ are required. The initial outflows 
for nodes with positive nominal demand, $d>0$ are set to mid-interval values, $c_{i}=d_{i} / 2$. The corresponding initial heads are given by the general formula $h_{i}^{(0)}=\left(h_{s}-h_{m}\right) \gamma^{-1}(1 / 2)+h_{m}+u_{i}$. This translates, for particular PORs, to $h_{i}^{(0)}=u_{i}+\left(h_{s}+a_{2} h_{m}\right) / a_{1}$ with (i) $a_{1}=2, a_{2}=1$ for the linear, cubic and logistic PORs; (ii) $a_{1}=3, a_{2}=2$ for the quadratic POR; and (iii) $a_{1}=4, a_{2}=3$ for the unregularized, 1-side regularized and 2-side regularized Wagner PORs

The initial flows for links which have no flow constraints are set (in SI units) to $q_{i}^{(0)}=$ $D_{i}^{2} \pi / 12$, (equivalent to a fluid velocity of $1 / 3 \mathrm{~m} / \mathrm{s}$ ) and the initial flows for links which have flow constraints are set to $\boldsymbol{q}^{(0)}=\left(\alpha \boldsymbol{q}_{\text {min }}+\beta \boldsymbol{q}_{\text {max }}\right) /(\alpha+\beta)$ with $\alpha=\beta=1$ for mid-interval initial link flows. Other choices of $\alpha$, and $\beta$ were also investigated and the results are reported below.

The initial Lagrange multipliers are set to $\lambda_{i}^{(0)}=\mu_{i}^{(0)}=\kappa_{i}^{(0)}=\nu_{i}^{(0)}=0, \forall i$ and all Lagrange multipliers are zeroed at the beginning of each iteration.

With $c_{i} / d_{i}=1 / 2$ and $\alpha=\beta=1$ the corresponding initial index sets are $\mathcal{I}_{q_{b}}=\left\{1,2, \ldots, n_{p}\right\}$, $\mathcal{I}_{c_{b}}=\left\{1,2, \ldots, n_{j}\right\}, \mathcal{I}_{c_{u}}=\mathcal{I}_{c_{l}}=\mathcal{I}_{q_{u}}=\mathcal{I}_{q_{l}}=\emptyset$. Note that where there are LFECs the initial set assignments of the corresponding link indices will be to either $\mathcal{I}_{q_{l}}$ or $\mathcal{I}_{q_{u}}$ according to the random choice made. These starting values may not be in the feasible set but provided the LP confirms the existence of a solution this is not a problem because mass balance will be satisfied at the solution.

\section{Regularizing the Schur complement}

It is possible that the Schur complement in (22) becomes singular. When this happens at a point which is away from the solution point, failure of the method can be avoided by regularizing the Schur complement. This does not affect the generally quadratic convergence of the procedure unless there is degeneracy at the solution point. Regularization would usually only be necessary if there are constraints applied to links in the network's spanning tree since this can lead to a violation of the LICQ: a phenomenon which cannot occur if constraints are only applied to co-tree links.

One simple heuristic regularization that has succeeded in the experience of the authors, and represents a variation on the Levenberg-Marquardt method (LMM), is to replace any of those zeros on the diagonal of the Schur complement by non-zeros. The choice of regularization parameter value can be varied adaptively, as is done in the LMM, if convergence remains slow but regularization will usually only be necessary until the iterates move away from the region where the Schur complement is singular or has large condition number. In many cases a value of 1 is sufficient but larger regularization parameters may be necessary and indeed were used in a few of the tests reported later which used the unregularized Wagner POR. The technique works well in the experience of the authors. A full Levenberg-Marquardt regularization scheme 
may address more difficult cases where matrix conditioning arrests or delays convergence but the investigation of this is beyond the scope of this paper.

\section{The iteration loop}

The iteration loop which implements the ASMFC is now described.

Start loop: For $m=0,1,2, \ldots$ repeat steps (a) to (l) until the stopping test is satisfied

(a) Zero all the Lagrange multipliers.

(b) Compute $\boldsymbol{M}_{c_{b}}^{(m)}=\operatorname{diag}\left\{h^{\prime}\left(c_{i_{1}}\right), h^{\prime}\left(c_{i_{2}}\right), \ldots, h^{\prime}\left(c_{i_{n_{b}}}\right)\right\}, i_{k} \in \mathcal{I}_{c_{b}}^{(m)}, 1 \leq k \leq n_{c_{b}}^{(m)}, \boldsymbol{V}_{b}^{(m)}$ of (22) and the right-hand-side of (23).

(c) Solve (23) for the corrections $\boldsymbol{h}^{(m+1)}-\boldsymbol{h}^{(m)}$ and use the corrections to update $\boldsymbol{h}^{(m+1)}$.

(d) Use (24) to update the link flows $q_{i}^{(m+1)}$ for $i \in \mathcal{I}_{q_{b}}$

(e) Use (25) to update the nodal outflows $c_{i}^{(m+1)}$ for which $i \in \mathcal{I}_{c_{b}}$.

(f) Use (26) to update the lower bound nodal outflow constraint Lagrange multipliers $\boldsymbol{\lambda}^{(m)}$ for the nodes with index $i \in \mathcal{I}_{c_{l}}$.

(g) Use (26) to update the upper bound nodal outflow constraint Lagrange multipliers, $\boldsymbol{\mu}^{(m)}$ for the nodes with index $i \in \mathcal{I}_{c_{u}}$.

(h) Use (27) to update the lower bound link flow constraint Lagrange multipliers $\boldsymbol{\kappa}^{(m)}$ for the links with index $i \in \mathcal{I}_{q_{l}}$.

(i) Use (28) to update the upper bound link flow constraint Lagrange multipliers $\boldsymbol{\nu}^{(m)}$ for the links with index $i \in \mathcal{I}_{q_{u}}$.

(j) Assign those links and nodes with unequal upper and lower bound constraints to the index sets $\mathcal{I}_{q_{b}}, \mathcal{I}_{q_{l}}, \mathcal{I}_{q_{u}}, \mathcal{I}_{c_{b}}, \mathcal{I}_{c_{l}}$, and $\mathcal{I}_{c_{u}}$. Then assign, if there are any, the LFEC links to the appropriate sets using the scheme described earlier.

(k) Project the exterior link flows onto the constraint boundaries $\boldsymbol{q}^{(m+1)} \leftarrow \max \left(\boldsymbol{q}^{(m+1)}, \boldsymbol{q}_{\min }\right)$ and then $\boldsymbol{q}^{(m+1)} \leftarrow \min \left(\boldsymbol{q}^{(m+1)}, \boldsymbol{q}_{\max }\right)$

(1) Project the exterior outflows onto the constraint boundaries $\boldsymbol{c}^{(m+1)} \leftarrow \max \left(\boldsymbol{c}^{(m+1)}, 0\right)$, $\boldsymbol{c}^{(m+1)} \leftarrow \min \left(\boldsymbol{c}^{(m+1)}, \boldsymbol{d}\right)$ 


\section{ILLUSTRATIVE EXAMPLES}

In this section the ASMFC is demonstrated on the illustrative network shown in Fig. 2. The constrained links are not confined to the cotree and this leaves open the possibility that zero flows or active constraints in some links could isolate parts of the network and thereby violate the LICQ.

The basic parameters of the network are given in Table 1. The steady-state solution shown is for the (unregularized) Wagner POR, a source head of $250 \mathrm{~m}$, minimum pressure head, $h_{m}=0 \mathrm{~m}$, and a service pressure head, $h_{s}=20 \mathrm{~m}$. All nodes have an elevation of $100 \mathrm{~m}$. Also shown in Fig. 2 are the nominal demands, $d$, the solution heads, $h$, flows $q$, delivery fractions as percentages of the nominal demands and the constraint upper and lower limits (shown in parentheses). Thus, links 2-10 have flow constraints and of these links 5, 7 and 8 have LFECs. All initial values were shown to be inside the feasible set.

The iterations were run until the relative differences between successive iterates

$$
\delta_{h}^{(m+1)}=\frac{\left\|\boldsymbol{h}^{(m+1)}-\boldsymbol{h}^{(m)}\right\|_{\infty}}{1+\left\|\boldsymbol{h}^{(m+1)}\right\|_{\infty}}, \quad \delta_{q}^{(m+1)}=\frac{\left\|\boldsymbol{q}^{(m+1)}-\boldsymbol{q}^{(m)}\right\|_{\infty}}{1+\left\|\boldsymbol{q}^{(m+1)}\right\|_{\infty}}, \quad \delta_{c}^{(m+1)}=\frac{\left\|\boldsymbol{c}_{b}^{(m+1)}-\boldsymbol{c}_{b}^{(m)}\right\|_{\infty}}{1+\left\|\boldsymbol{c}_{b}^{(m+1)}\right\|_{\infty}}
$$

were smaller than the prescribed stopping tolerance, $10^{-10}$. The choice $\alpha=\beta=1$ took 6 iterations to satisfy the stopping test, the choice $\alpha=0, \beta=1$ took 7 iterations and the choice $\alpha=1, \beta=0$ took 11 iterations. Extensive tests were conducted with different values of $\alpha$ and $\beta$ on this and other networks with link flow constraints but in the experience of the authors the values $\alpha=\beta=1$ most often took the fewest iterations. The convergence data show the typical quadratic convergence associated with Newton's method. It is worth noting that the derivative assignment technique described in Deuerlein et al. (2019) was necessary in the solution of this network (because the derivative of the Wagner POR is undefined at $h=h_{m}$ ). It was also necessary to invoke the Schur complement regularization scheme described above to counteract the singularity of the Schur complement during one of the early iterations.

At steady-state (i) the set memberships are: $\mathcal{I}_{q_{b}}=\{1,9,10\}, \mathcal{I}_{q_{u}}=\{2,3,4,5,8\}, \mathcal{I}_{q_{l}}=$ $\{6,7\}, \mathcal{I}_{c_{b}}=\{3,4,5,6,7,8\}$ and $\mathcal{I}_{c_{u}}=\{1\}, \mathcal{I}_{c_{l}}=\{2\}$ (ii) the KKT conditions were satisfied and (iii) the LICQ condition was not violated. The steady-state energy, mass and outflow residuals all had norm smaller that $10^{-13}$, a value consistent with the fact that all calculations (including those for other examples in this paper) were performed using Matlab (Mathworks 2016) which uses a floating point number system that conforms with IEEE standard double precision arithmetic (IEEE 2008) for which machine epsilon is about $2.2 \times 10^{-16}$.

It is seen that the flow constraints on links 9 and 10 are inactive and the sum of their flows 
into node 2 exactly cancels the fixed flow in link 8 thereby delivering zero outflow to node 2 . The flow in link 6 is at the lower link flow constraint boundary and, since the corresponding multiplier in this case can be interpreted as a negative head loss, this constraint can be seen as acting as a pump. Similarly, the flow in link 8 has direction opposite to that of the unconstrained flow and so that too can be seen as acting as a pump.

The ASMFC was also applied to eight case study networks $N_{1}-N_{8}$ (four of which are available online in the ASCE Library (www.ascelibrary.org) which have between 934 and 19,647 links and between 848 and 17,971 nodes, to demonstrate the effectiveness of the method on realistic problems. Each of the eight networks had 60 cotree link flow constraints: 57 links were flowconstrained to $10 \%$ of that link's unconstrained steady-state PDM flow and another 3 had LFEC. This has the effect of simulating 60 flow control valves in each network.

In all cases (i) the nominal demands were magnified, in repeated tests, by factors of 5, 20 and 40 to assess the behaviour of the method in solving increasingly difficult PDM problems (ii) the 1-side regularized Wagner POR was used (iii) the mid-interval starting schemes described above were used and (iv) the values $\alpha=\beta=1$ were used in the starting values formula and (v) the stopping tolerance for the infinity norms of the relative differences between successive iterates was set at $10^{-10}$. This stopping tolerance (even though it is smaller than would normally be used in practice) was chosen to ensure that the quadratic convergence normally associated with Newton's method was evident.

Columns 2, 3 and 4 of Table 2 show the number of links, nodes and sources for the eight case study networks. The other columns of Table 2 show the convergence data for the application of the method to the case study network $N_{1}-N_{8}$ with a demand magnification factor $f=5$ : column 5 shows the number, $m$, of iterations to satisfy the stopping test, column 6 shows the delivery fraction, $\zeta$, as a percentage of the nominal demand, columns 7-9 show the quantities $\tau_{q, h, c}=-\log _{10}\left(\delta_{q, h, c}^{(m)}\right)$ which represent the numbers of decimal digits of agreement between the two last iterates for the link flows, heads and outflows, respectively, and columns 10-12 show the accuracy of the final energy, mass and outflow residuals shown as the negative base-10 logarithms of the residual norms themselves. All these results are consistent with a stopping test based on a relative norm difference no greater than $10^{-10}$. The corresponding convergence results for the other networks, other PORs and the other demand magnification factors are remarkably similar to those in Table 2.

In runs on all the case study networks and with all 3 demand magnification factors, the method took at best 9 and at most 13 iterations to satisfy the stopping test based on the measures in (29). The mean of the number of iterations was 11.6 with a standard deviation of 1.2. With the starting values $\alpha=0, \beta=1$ and $\alpha=1, \beta=0$ all cases converged in no more than 14 iterations and the quadratic convergence was evident in all cases. The behaviour of the 
method for these problems with, for example, the linear or the unregularized Wagner PORs was almost identical with the behaviour described so far although the delivery fractions were, naturally, different for the other PORs .

The derivative assignment technique described in Deuerlein et al. (2019), which is part of the implementation code for these tests was automatically invoked for the unregularized Wagner POR to overcome the difficulties created by the fact that the derivative of that POR is undefined when $h=h_{m}=0$. Where the (unreasonable) demand magnification factor of 40 was used to engineer a more difficult problem, a larger Schur complement regularization parameter was, in 2 cases, necessary to ensure convergence.

\section{CONCLUSIONS}

A new content-based active set method, ASMFC, for the determination of link flows, nodal heads and outflows in a PDM WDS which has link flow constraints is presented. The method extends the authors' previous work which was an active set method for the determination of link flows, nodal heads and outflows in a PDM WDS with unconstrained link flows. The necessary and sufficient KKT conditions for a minimum of the content function optimization problem are given and the Newton method based on this formulation leads, after some algebraic simplifications, to the ASMFC. For this model the non-existence of a solution can result only when the (wrong) choice of constraints has the consequence that the polyhedral feasible set which is composed of (i) the continuity equation, (ii) the flow constraints and (iii) the outflow conditions, is empty. Whether or not the feasible set is empty can be easily tested using a linear program. The uniqueness of the solution to the problem is assured provided the LICQ is not violated at the solution.

FCVs and pumps can be modelled and heuristics are not needed to determine the status of control devices in the system: their states are found as part of the solution. Choosing equal upper and lower bound constraints (LFEC) is equivalent to specifying a fixed link flow rate and is easily handled by the method. The range of models for which such solutions exist is greater than that for DDM problems by virtue of the relaxation of demand constraints in PDM modeling. There is no risk of isolated demands in this case since in PDM problems the nodal outflow can reduce to zero if the pressure is insufficient.

The operation of the ASMFC is illustrated on a small example network and it's efficacy is demonstrated by applying it to eight case study networks with between 934 and 19,647 links and between 848 and 17,971 nodes. The case study networks each had 60 cotree link flow constraints which either (i) limit the maximum flow in a link to $10 \%$ of its unconstrained value or (ii) prescribe a LFEC. The method found, for various tested starting sets, the steady-state solutions, even on the largest case study network, in fewer than 14 iterations. The quadratic 
convergence usually associated with Newton's method was evident in all cases. A regularization of the Schur complement may be necessary in some cases. The new method is applicable to all the PORs for which the ASM of Deuerlein et al. (2019) is applicable provided the special methods introduced there to account for irregularities in the inverse POR are invoked.

The ASMFC method has application in real-time operations. The operation of drinking water distribution systems is becoming more and more complex for a variety of reasons. For instance, as events in recent years have shown, even in Europe the availability of the water as a resource is not guaranteed in every region during the increasingly hot and dry summer periods. As a consequence water suppliers are forced to develop alternative resources that may be shared by different supply utilities. This means that there is a rapidly growing need for enhanced system operations such as control of the flow from different sources. In a worst-case scenario where the available resources are not sufficient to cover the demand, flow control is also an indispensable tool for efficient distribution of the available water.

Improved simulation techniques are also required that are able to deal with new developments in the management of WDSs. An invaluable tool for operation of such systems is a near real-time hydraulic solver that solves the control equations and may be integrated within newly developed IOT platforms in the future. The ASMFC solver is such a tool.

In addition to real-time simulations ASMFC also has application in the optimal design of future new supply systems and their control strategies. The ASMFC has, for example, application in pump system design: for a given positive lower flow bound the solver calculates the required pumping head that is necessary to maintain that desired flow. This technique can be used in system design as well as in control optimization.

Last, but not least, stable solution methods for systems under control conditions form the basis of all optimization applications or Monte Carlo simulations that require a huge number of simulation runs with a great variety of boundary conditions (that may have a strong impact on the operation of flow control devices). Future research will be focussed on the integration of pressure control devices and pumps. 
APPENDIX: Analytical separation of the system, LICQ

\section{ANALYTICAL SEPARATION}

Certain unknowns in the system (20) can, by exploiting the structure of the system, be expressed analytically in terms of other parts and this analytical separation helps to clarify the exposition and to simplify the algorithmic implementation of the ASMFC. This separation process is now described.

The 12-th block row of $(20)$ is $-\boldsymbol{I}_{q l}\left(\boldsymbol{q}_{l}^{(m+1)}-\boldsymbol{q}_{l}^{(m)}\right)=\boldsymbol{q}_{l}^{(m)}-\boldsymbol{q}_{l, \text { min }}$ from which it is evident that $\boldsymbol{q}_{l}^{(m+1)}=\boldsymbol{q}_{l, \text { min }}$, where $\boldsymbol{q}_{l, \text { min }}$ is the vector of constraint lower bounds for the links in $\mathcal{I}_{q_{l}}$. By similar reasoning the 13-th block row of (20) gives $\boldsymbol{q}_{u}^{(m+1)}=\boldsymbol{q}_{u, \text { max }}$. Substituting these into (20) gives the reduced, $11 \times 11$ equivalent system (with block columns 2 and 3 removed and block rows 12 and 13 of (20) removed) where

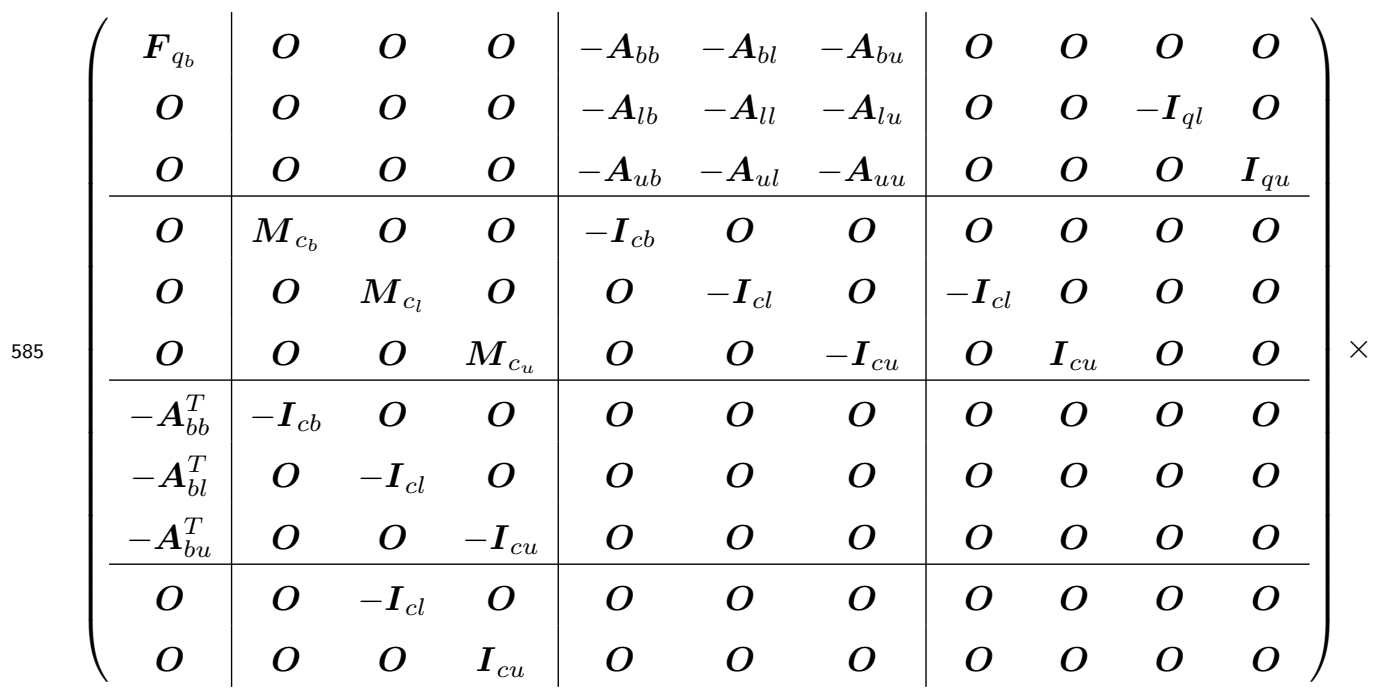

$586 \quad\left(\begin{array}{c}\boldsymbol{q}_{b}^{(m+1)}-\boldsymbol{q}_{b}^{(m)} \\ \hline \boldsymbol{c}_{b}^{(m+1)}-\boldsymbol{c}_{b}^{(m)} \\ \boldsymbol{c}_{l}^{(m+1)}-\boldsymbol{c}_{l}^{(m)} \\ \boldsymbol{c}_{u}^{(m+1)}-\boldsymbol{c}_{u}^{(m)} \\ \hline \boldsymbol{h}_{c_{b}}^{(m+1)}-\boldsymbol{h}_{c_{b}}^{(m)} \\ \boldsymbol{h}_{c_{l}}^{(m+1)}-\boldsymbol{h}_{c_{l}}^{(m)} \\ \boldsymbol{h}_{c_{u}}^{(m+1)}-\boldsymbol{h}_{c_{u}}^{(m)} \\ \boldsymbol{\lambda}^{(m+1)}-\boldsymbol{\lambda}^{(m)} \\ \boldsymbol{\mu}^{(m+1)}-\boldsymbol{\mu}^{(m)} \\ \boldsymbol{\kappa}^{(m+1)}-\boldsymbol{\kappa}^{(m)} \\ \boldsymbol{\nu}^{(m+1)}-\boldsymbol{\nu}^{(m)}\end{array}\right)=-\left(\begin{array}{c}\boldsymbol{G}_{q_{b}} \boldsymbol{q}_{b}^{(m)}-\boldsymbol{A}_{b b} \boldsymbol{h}_{c_{b}}^{(m)}-\boldsymbol{A}_{b l} \boldsymbol{h}_{c_{l}}^{(m)}-\boldsymbol{A}_{b u} \boldsymbol{h}_{c_{u}}^{(m)}-\boldsymbol{a}_{q_{b}} \\ \boldsymbol{G}_{q_{l}} \boldsymbol{q}_{l}^{(m)}-\boldsymbol{A}_{l b} \boldsymbol{h}_{c_{b}}^{(m)}-\boldsymbol{A}_{l l} \boldsymbol{h}_{c_{l}}^{(m)}-\boldsymbol{A}_{l u} \boldsymbol{h}_{c_{u}}^{(m)}-\boldsymbol{a}_{q_{l}}-\boldsymbol{\kappa}^{(m)}+\boldsymbol{F}_{q_{l}}\left(\boldsymbol{q}_{l, m i n}-\boldsymbol{q}_{l}^{(m)}\right) \\ \boldsymbol{G}_{q_{u}} \boldsymbol{q}_{u}^{(m)}-\boldsymbol{A}_{u b} \boldsymbol{h}_{c_{b}}^{(m)}-\boldsymbol{A}_{u l} \boldsymbol{h}_{c_{l}}^{(m)}-\boldsymbol{A}_{u u} \boldsymbol{h}_{c_{u}}^{(m)}-\boldsymbol{a}_{q_{u}}+\boldsymbol{\nu}^{(m)}+\boldsymbol{F}_{q_{u}}\left(\boldsymbol{q}_{u, m a x}-\boldsymbol{q}_{u}^{(m)}\right) \\ \boldsymbol{h}\left(\boldsymbol{c}_{b}^{(m)}\right)-\boldsymbol{h}_{c_{b}}^{(m)} \\ -\boldsymbol{h}_{c_{l}}^{(m)}+h_{m}+\boldsymbol{u}_{c_{l}}-\boldsymbol{\lambda}^{(m)} \\ -\boldsymbol{h}_{c_{u}}^{(m)}+h_{s}+\boldsymbol{u}_{c_{u}}+\boldsymbol{\mu}^{(m)} \\ -\boldsymbol{A}_{b b}^{T} \boldsymbol{q}_{b}^{(m)}-\boldsymbol{A}_{l b}^{T} \boldsymbol{q}_{l, m i n}-\boldsymbol{A}_{u b}^{T} \boldsymbol{q}_{u, \text { max }}-\boldsymbol{c}_{b}^{(m)} \\ -\boldsymbol{A}_{b l}^{T} \boldsymbol{q}_{b}^{(m)}-\boldsymbol{A}_{l l}^{T} \boldsymbol{q}_{l, \min }-\boldsymbol{A}_{u l}^{T} \boldsymbol{q}_{u, \text { max }}-\boldsymbol{c}_{l}^{(m)} \\ -\boldsymbol{A}_{b u}^{T} \boldsymbol{q}_{b}^{(m)}-\boldsymbol{A}_{l u}^{T} \boldsymbol{q}_{l, m i n}-\boldsymbol{A}_{u u}^{T} \boldsymbol{q}_{u, \max }-\boldsymbol{c}_{u}^{(m)} \\ -\boldsymbol{c}_{l}^{(m)} \\ \boldsymbol{c}_{u}^{(m)}-\boldsymbol{d}_{u}\end{array}\right)$


${ }_{587}$ The 10-th block equation in this system leads to $\boldsymbol{c}_{l}^{(m+1)}=0$ and the 11-th leads to $\boldsymbol{c}_{u}^{(m+1)}=\boldsymbol{d}_{u}$

from which it follows that the reduced system (with block columns 3 and 4 and block rows 10 and 11 removed) can be written
$590\left(\begin{array}{c|c|ccc|cccc}\boldsymbol{F}_{q b} & \boldsymbol{O} & -\boldsymbol{A}_{b b} & -\boldsymbol{A}_{b l} & -\boldsymbol{A}_{b u} & \boldsymbol{O} & \boldsymbol{O} & \boldsymbol{O} & \boldsymbol{O} \\ \boldsymbol{O} & \boldsymbol{O} & -\boldsymbol{A}_{l b} & -\boldsymbol{A}_{l l} & -\boldsymbol{A}_{l u} & \boldsymbol{O} & \boldsymbol{O} & -\boldsymbol{I}_{q l} & \boldsymbol{O} \\ \boldsymbol{O} & \boldsymbol{O} & -\boldsymbol{A}_{u b} & -\boldsymbol{A}_{u l} & -\boldsymbol{A}_{u u} & \boldsymbol{O} & \boldsymbol{O} & \boldsymbol{O} & \boldsymbol{I}_{q u} \\ \hline \boldsymbol{O} & \boldsymbol{M}_{c b} & -\boldsymbol{I}_{c b} & \boldsymbol{O} & \boldsymbol{O} & \boldsymbol{O} & \boldsymbol{O} & \boldsymbol{O} & \boldsymbol{O} \\ \boldsymbol{O} & \boldsymbol{O} & \boldsymbol{O} & -\boldsymbol{I}_{c l} & \boldsymbol{O} & -\boldsymbol{I}_{c l} & \boldsymbol{O} & \boldsymbol{O} & \boldsymbol{O} \\ \boldsymbol{O} & \boldsymbol{O} & \boldsymbol{O} & \boldsymbol{O} & -\boldsymbol{I}_{c u} & \boldsymbol{O} & \boldsymbol{I}_{c u} & \boldsymbol{O} & \boldsymbol{O} \\ \hline-\boldsymbol{A}_{b b}^{T} & -\boldsymbol{I}_{c b} & \boldsymbol{O} & \boldsymbol{O} & \boldsymbol{O} & \boldsymbol{O} & \boldsymbol{O} & \boldsymbol{O} & \boldsymbol{O} \\ -\boldsymbol{A}_{b l}^{T} & \boldsymbol{O} & \boldsymbol{O} & \boldsymbol{O} & \boldsymbol{O} & \boldsymbol{O} & \boldsymbol{O} & \boldsymbol{O} & \boldsymbol{O} \\ -\boldsymbol{A}_{b u}^{T} & \boldsymbol{O} & \boldsymbol{O} & \boldsymbol{O} & \boldsymbol{O} & \boldsymbol{O} & \boldsymbol{O} & \boldsymbol{O} & \boldsymbol{O}\end{array}\right) \times$

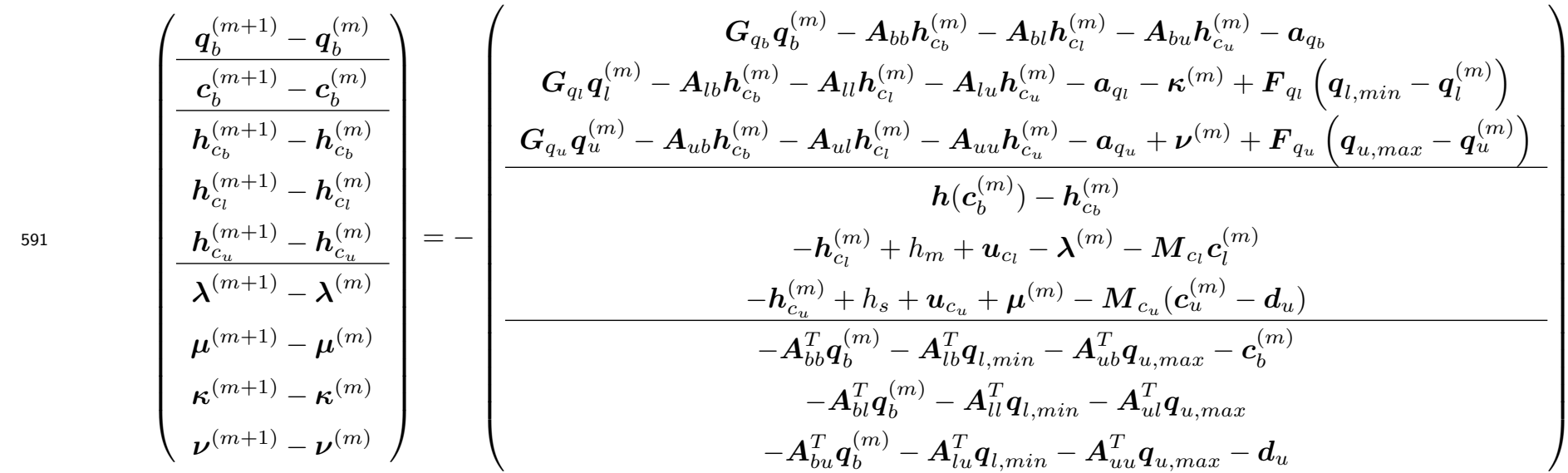

${ }_{592}$ Consider the submatrix of (30) made up of block rows $1,4,7,8,9$. This subsystem is independent of the last four unknowns and so can be written

$$
\left(\begin{array}{c|c|ccc|cccc}
\boldsymbol{F}_{q_{b}} & \boldsymbol{O} & -\boldsymbol{A}_{b b} & -\boldsymbol{A}_{b l} & -\boldsymbol{A}_{b u} & \boldsymbol{O} & \boldsymbol{O} & O & \boldsymbol{O} \\
\hline \boldsymbol{O} & \boldsymbol{M}_{c_{b}} & -\boldsymbol{I}_{c b} & O & O & O & O & O & O \\
\hline-\boldsymbol{A}_{b b}^{T} & -\boldsymbol{I}_{c b} & O & O & O & O & O & O & O \\
-\boldsymbol{A}_{b l}^{T} & O & O & O & O & O & O & O & O \\
-\boldsymbol{A}_{b u}^{T} & O & O & O & O & O & O & O & O
\end{array}\right) \times
$$




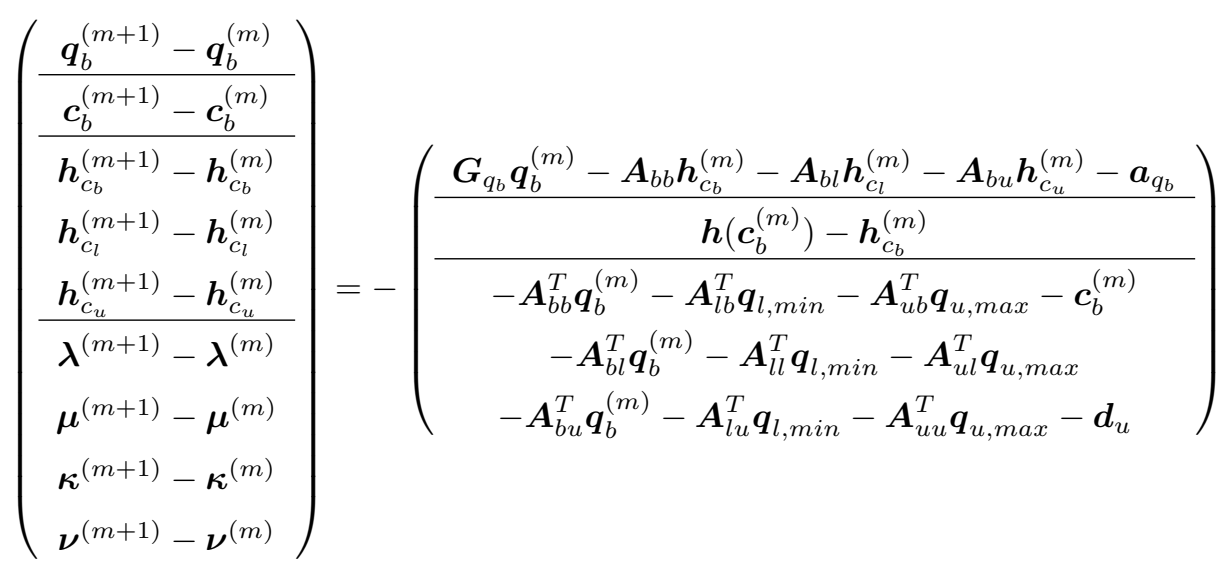

603

604

605

606

607

608

609

610

611

612

98

or more simply as

$$
\left(\begin{array}{c|c|ccc}
\boldsymbol{F}_{q_{b}} & \boldsymbol{O} & -\boldsymbol{A}_{b b} & -\boldsymbol{A}_{b l} & -\boldsymbol{A}_{b u} \\
\hline \boldsymbol{O} & \boldsymbol{M}_{c_{b}} & -\boldsymbol{I}_{c b} & \boldsymbol{O} & \boldsymbol{O} \\
\hline-\boldsymbol{A}_{b b}^{T} & -\boldsymbol{I}_{c b} & \boldsymbol{O} & \boldsymbol{O} & \boldsymbol{O} \\
-\boldsymbol{A}_{b l}^{T} & \boldsymbol{O} & \boldsymbol{O} & \boldsymbol{O} & \boldsymbol{O} \\
-\boldsymbol{A}_{b u}^{T} & \boldsymbol{O} & \boldsymbol{O} & \boldsymbol{O} & \boldsymbol{O}
\end{array}\right)\left(\begin{array}{c}
\boldsymbol{q}_{b}^{(m+1)}-\boldsymbol{q}_{b}^{(m)} \\
\frac{\boldsymbol{c}_{b}^{(m+1)}-\boldsymbol{c}_{b}^{(m)}}{\boldsymbol{h}_{c_{b}}^{(m+1)}-\boldsymbol{h}_{c_{b}}^{(m)}} \\
\boldsymbol{h}_{c_{l}}^{(m+1)}-\boldsymbol{h}_{c_{l}}^{(m)} \\
\boldsymbol{h}_{c_{u}}^{(m+1)}-\boldsymbol{h}_{c_{u}}^{(m)}
\end{array}\right)=
$$

$$
-\left(\begin{array}{c}
\frac{\boldsymbol{G}_{q_{b}} \boldsymbol{q}_{b}^{(m)}-\boldsymbol{A}_{b b} \boldsymbol{h}_{c_{b}}^{(m)}-\boldsymbol{A}_{b l} \boldsymbol{h}_{c_{l}}^{(m)}-\boldsymbol{A}_{b u} \boldsymbol{h}_{c_{u}}^{(m)}-\boldsymbol{a}_{q_{b}}}{\boldsymbol{h}\left(\boldsymbol{c}_{b}^{(m)}\right)-\boldsymbol{h}_{c_{b}}^{(m)}} \\
-\boldsymbol{A}_{b b}^{T} \boldsymbol{q}_{b}^{(m)}-\boldsymbol{A}_{l b}^{T} \boldsymbol{q}_{l, \min }-\boldsymbol{A}_{u b}^{T} \boldsymbol{q}_{u, \max }-\boldsymbol{c}_{b}^{(m)} \\
-\boldsymbol{A}_{b l}^{T} \boldsymbol{q}_{b}^{(m)}-\boldsymbol{A}_{l l}^{T} \boldsymbol{q}_{l, \min }-\boldsymbol{A}_{u l}^{T} \boldsymbol{q}_{u, \max } \\
-\boldsymbol{A}_{b u}^{T} \boldsymbol{q}_{b}^{(m)}-\boldsymbol{A}_{l u}^{T} \boldsymbol{q}_{l, \text { min }}-\boldsymbol{A}_{u u}^{T} \boldsymbol{q}_{u, \max }-\boldsymbol{d}_{u}
\end{array}\right) .
$$

Once the unknowns in (31) have been found, the Lagrange multipliers can be found from block equations 2,3,5, and 6 of (31) which can be rewritten, after some manipulations as

$$
\begin{gathered}
\boldsymbol{\lambda}^{(m+1)}=h_{m}+\boldsymbol{u}_{c_{l}}-\boldsymbol{h}_{c_{l}}^{(m+1)}-\boldsymbol{M}_{c_{l}} \boldsymbol{c}_{l}^{(m)} \\
\boldsymbol{\mu}^{(m+1)}=\boldsymbol{h}_{c_{u}}^{(m+1)}-h_{s}-\boldsymbol{u}_{c_{u}}+\boldsymbol{M}_{c_{u}}\left(\boldsymbol{c}_{u}^{(m)}-\boldsymbol{d}_{u}\right) \\
\boldsymbol{\kappa}^{(m+1)}=\boldsymbol{G}_{q_{l}} \boldsymbol{q}_{l}^{(m)}-\boldsymbol{A}_{l b} \boldsymbol{h}_{c_{b}}^{(m+1)}-\boldsymbol{A}_{l l} \boldsymbol{h}_{c_{l}}^{(m+1)}-\boldsymbol{A}_{l u} \boldsymbol{h}_{c_{u}}^{(m+1)}-\boldsymbol{a}_{q_{l}}+\boldsymbol{F}_{q_{l}}\left(\boldsymbol{q}_{l, m i n}-\boldsymbol{q}_{l}^{(m)}\right) \\
\boldsymbol{\nu}^{(m+1)}=-\left(\boldsymbol{G}_{q_{u}} \boldsymbol{q}_{u}^{(m)}-\boldsymbol{A}_{u b} \boldsymbol{h}_{c_{b}}^{(m+1)}-\boldsymbol{A}_{u l} \boldsymbol{h}_{c_{l}}^{(m+1)}-\boldsymbol{A}_{u u} \boldsymbol{h}_{c_{u}}^{(m+1)}-\boldsymbol{a}_{q_{u}}+\boldsymbol{F}_{q_{u}}\left(\boldsymbol{q}_{u, \text { max }}-\boldsymbol{q}_{u}^{(m)}\right)\right)
\end{gathered}
$$

Omitting the last terms in parentheses, which vanish by definition, gives

$$
\begin{gathered}
\boldsymbol{\lambda}^{(m+1)}=h_{m}+\boldsymbol{u}_{c_{l}}-\boldsymbol{h}_{c_{l}}^{(m+1)} \\
\boldsymbol{\mu}^{(m+1)}=\boldsymbol{h}_{c_{u}}^{(m+1)}-h_{s}-\boldsymbol{u}_{c_{u}}
\end{gathered}
$$




$$
\begin{gathered}
\boldsymbol{\kappa}^{(m+1)}=\boldsymbol{G}_{q_{l}} \boldsymbol{q}_{l}^{(m)}-\boldsymbol{A}_{l b} \boldsymbol{h}_{c_{b}}^{(m+1)}-\boldsymbol{A}_{l l} \boldsymbol{h}_{c_{l}}^{(m+1)}-\boldsymbol{A}_{l u} \boldsymbol{h}_{c_{u}}^{(m+1)}-\boldsymbol{a}_{q_{l}} \\
\boldsymbol{\nu}^{(m+1)}=-\left(\boldsymbol{G}_{q_{u}} \boldsymbol{q}_{u}^{(m)}-\boldsymbol{A}_{u b} \boldsymbol{h}_{c_{b}}^{(m+1)}-\boldsymbol{A}_{u l} \boldsymbol{h}_{c_{l}}^{(m+1)}-\boldsymbol{A}_{u u} \boldsymbol{h}_{c_{u}}^{(m+1)}-\boldsymbol{a}_{q_{u}}\right)
\end{gathered}
$$

if we denote, $\widehat{\boldsymbol{I}}_{c b}=\left(\begin{array}{lll}\boldsymbol{I}_{c b} & \boldsymbol{O} & \boldsymbol{O}\end{array}\right)$ and $\widehat{\boldsymbol{I}}_{c u}=\left(\begin{array}{lll}\boldsymbol{O} & \boldsymbol{O} & \boldsymbol{I}_{c u}\end{array}\right)$ the first block row of

$$
\left(\begin{array}{c}
\boldsymbol{q}_{b}^{(m+1)}-\boldsymbol{q}_{b}^{(m)} \\
\boldsymbol{c}_{b}^{(m+1)}-\boldsymbol{c}_{b}^{(m)}
\end{array}\right)=\left(\begin{array}{cc}
\boldsymbol{F}_{q_{b}}^{-1} & \boldsymbol{O} \\
\boldsymbol{O} & \boldsymbol{M}_{c_{b}}^{-1}
\end{array}\right)\left[-\left(\begin{array}{c}
\boldsymbol{G}_{q_{b}} \boldsymbol{q}_{b}^{(m)}-\boldsymbol{A}_{b .} \boldsymbol{h}^{(m)}-\boldsymbol{a}_{q_{b}} \\
\boldsymbol{h}\left(\boldsymbol{c}_{b}^{(m)}\right)-\boldsymbol{h}_{c_{b}}^{(m)}
\end{array}\right)+\left(\begin{array}{c}
\boldsymbol{A}_{b .} \\
\widehat{\boldsymbol{I}}_{c b}
\end{array}\right)\left(\boldsymbol{h}^{(m+1)}-\boldsymbol{h}^{(m)}\right)\right]
$$

is

$$
\begin{aligned}
\left(\boldsymbol{q}_{b}^{(m+1)}-\boldsymbol{q}_{b}^{(m)}\right) & =-\boldsymbol{F}_{q_{b}}^{-1}\left(\boldsymbol{G}_{q_{b}} \boldsymbol{q}_{b}^{(m)}-\boldsymbol{A}_{b .} \boldsymbol{h}^{(m)}-\boldsymbol{a}_{q_{b}}-\boldsymbol{A}_{b .}\left(\boldsymbol{h}^{(m+1)}-\boldsymbol{h}^{(m)}\right)\right) \\
& =-\boldsymbol{F}_{q_{b}}^{-1}\left(\boldsymbol{G}_{q_{b}} \boldsymbol{q}_{b}^{(m)}-\boldsymbol{A}_{b .} \boldsymbol{h}^{(m+1)}-\boldsymbol{a}_{q_{b}}\right)
\end{aligned}
$$

or

$$
\boldsymbol{q}_{b}^{(m+1)}=\boldsymbol{q}_{b}^{(m)}-\boldsymbol{F}_{q_{b}}^{-1}\left(\boldsymbol{G}_{q_{b}} \boldsymbol{q}_{b}^{(m)}-\boldsymbol{A}_{b .} \boldsymbol{h}^{(m+1)}-\boldsymbol{a}_{q_{b}}\right)
$$

and the second block row is

$$
\begin{aligned}
\boldsymbol{c}_{b}^{(m+1)} & =\boldsymbol{c}_{b}^{(m)}-\boldsymbol{M}_{c_{b}}^{-1}\left(\boldsymbol{h}\left(\boldsymbol{c}_{b}^{(m)}\right)-\boldsymbol{h}_{c_{b}}^{(m)}-\widehat{\boldsymbol{I}}_{c b}\left(\boldsymbol{h}^{(m+1)}-\boldsymbol{h}^{(m)}\right)\right) \\
& =\boldsymbol{c}_{b}^{(m)}-\boldsymbol{M}_{c_{b}}^{-1}\left(\boldsymbol{h}\left(\boldsymbol{c}_{b}^{(m)}\right)-\boldsymbol{h}_{c_{b}}^{(m)}-\left(\boldsymbol{h}_{c_{b}}^{(m+1)}-\boldsymbol{h}_{c_{b}}^{(m)}\right)\right) \\
& =\boldsymbol{c}_{b}^{(m)}-\boldsymbol{M}_{c_{b}}^{-1}\left(\boldsymbol{h}\left(\boldsymbol{c}_{b}^{(m)}\right)-\boldsymbol{h}_{c_{b}}^{(m+1)}\right) .
\end{aligned}
$$

\section{LINEAR INDEPENDENCE CONSTRAINT QUALIFICATION}

The inequality constraints in the system are $-c_{i} \leq 0, c_{i} \leq d_{i}, i=1,2, \ldots, n_{j} q_{j} \leq q_{\max , j}$, $-q_{j} \leq-q_{\text {min, }, j}, j=1,2, \ldots, n_{p}$. Suppose that certain of these inequality constraints are, at some point during the optimization process active, or binding: $c_{i_{1}}=0, c_{i_{2}}=d_{i_{2}}, i_{1}, i_{2} \in S$ and $q_{j_{1}}=q_{\max , j_{1}}, q_{j_{2}}=q_{\min , j_{2}}, j_{1}, j_{2} \in T, S$ and $T$, subsets of the index sets $\left\{1,2, \ldots, n_{j}\right\}$ and $\left\{1,2, \ldots, n_{p}\right\}$, respectively. In order for the Lagrange multipliers to be uniquely defined during the optimization process these binding constraints, together with the conservation of mass equations (another set of equality constraints) must form a set of linearly independent equations. More precisely, let $\boldsymbol{U}_{q_{l}}$ be a matrix made up of the rows of an $n_{q_{l}}$ identity which correspond to an active link flow lower constraint, $\boldsymbol{U}_{q_{u}}$ a matrix made up of the rows of an $n_{q_{u}}$ identity which correspond to an active link flow upper constraint, and let $\boldsymbol{U}_{c_{l}}$ be a matrix made up of the rows of an $n_{c_{l}}$ identity which correspond to an active outflow lower constraint, $\boldsymbol{U}_{c_{u}}$ a matrix made up of the rows of an $n_{c_{u}}$ identity which correspond to an active outflow upper constraint. Let $\boldsymbol{q}_{l, \text { min }} \in \mathbb{R}^{n_{q_{l}}}$ be a vector of the link flow lower constraint values of those 


$$
\boldsymbol{C}\left(\begin{array}{l}
\boldsymbol{q} \\
\boldsymbol{c}
\end{array}\right) \stackrel{n_{p}}{\stackrel{\text { def }}{=}} \begin{gathered}
n_{q_{l}} \\
n_{q_{u}} \\
n_{c_{l}} \\
n_{c_{u}}
\end{gathered}\left(\begin{array}{cc}
\boldsymbol{A}^{T} & \boldsymbol{I} \\
-\boldsymbol{U}_{q_{l}} & \\
\boldsymbol{U}_{q_{u}} & \\
& -\boldsymbol{U}_{c_{l}}
\end{array}\right)\left(\begin{array}{l}
\boldsymbol{q} \\
\boldsymbol{c}
\end{array}\right)=\left(\begin{array}{c}
\boldsymbol{o} \\
-\boldsymbol{q}_{l, \min } \\
\boldsymbol{q}_{u, \max } \\
\boldsymbol{o} \\
\boldsymbol{d}_{u}
\end{array}\right) .
$$

links for which the lower constraint is active and let $\boldsymbol{q}_{u, \max } \in \mathbb{R}^{n_{q_{u}}}$ be a vector of the link flow upper constraint values of those links for which the upper constraints are active. Let $\boldsymbol{d}_{u} \in \mathbb{R}^{n_{c u}}$ be a vector of the demands for nodes at which the upper outflow constraints are active. The general full equality constraint system is

(1)

The top block represents the equality constraints that make up the conservation of mass equations and are constant and the bottom four blocks represent the link flow and outflow constraints which are active and can change from one iteration to the next. Some, or all, of the lower four blocks may be empty at any given iteration. The LICQ is said to hold when the matrix $\boldsymbol{C}$ has full rank. The LICQ is necessary for the KKT conditions hold at a local minimizer.

\section{ACKNOWLEDGMENTS}

The work presented in this paper is part of the French-German collaborative research project ResiWater that is funded by the French National Research Agency (ANR; project: ANR-14PICS-0003) and the German Federal Ministry of Education and Research (BMBF; project: BMBF-13N13690).

\section{DATA AVAILABILITY}

The network shown in Fig. 2 and the networks $N_{1}, N_{3}, N_{4}$ and $N_{7}$, which are listed in Table 2, and their EPANET . inp files were previously published in Deuerlein et al. (2019). The other four networks $N_{2}, N_{5}, N_{6}$ and $N_{8}$ are not freely available either because they are proprietary or because of security concerns.

\section{References}

Alvarruiz, F., Alzamora, F. \& Vidal, A. M. (2018), 'Efficient modeling of active control valves in water distribution systems using the loop method', J. Hydraul. Eng. 144(10), 04018064. DOI: 10.1061/(ASCE)WR.1943-5452.0000982. 
Alvarruiz, F., Martnez-Alzamora, F. \& Vidal, A. M. (2015), 'Improving the efficiency of the loop method for the simulation of water distribution systems', J. Water Resour. Plann. Manage. 141(10), 04015019.

Boyd, S. P. \& Vandenberghe, L. (2009), Convex Optimization, 7th edn, Cambridge University Press, UK.

Collins, M., Cooper, L., Helgason, R., Kennington, J. \& LeBlanc, L. (1978), 'Solving the pipe network analysis problem using optimization techniques', Management Science 24(7), 747760.

Deuerlein, J., Piller, O., Elhay, S. \& Simpson, A. R. (2019), 'A content-based active set method for the pressure dependent model of water distribution systems', J. Water Resour. Plann. Manage. 145(1), 04018082. DOI: 10.1061/(ASCE)WR.1943-5452.0001003.

Deuerlein, J., Simpson, A. \& Dempe, S. (2009), 'Modeling the behavior of flow regulating devices in water distribution systems using constrained nonlinear programming', J. Hydraul. Eng. 135(11), 970-982.

Deuerlein, J., Simpson, A. R. \& Gross, E. (2008), The never-ending story of modeling control devices in hydraulic systems analysis, in J. E. V. Zyl, A. A. Ilemobade \& H. Jacobs, eds, 'Proceedings of the 10th Annual Water Distribution Systems Analysis Conference WDSA2008', Kruger National Park, South Africa.

Elhay, S., Piller, O., Deuerlein, J. W. \& Simpson, A. R. (2016), 'A robust, rapidly convergent method that solves the water distribution equations for pressure dependent models', $J$. Water Resour. Plann. Manage. 142(2). DOI: 10.1061/(ASCE)WR.1943-5452.0000578.

Golub, G. \& Van Loan, C. (1983), Matrix computations, North Oxford Academic Publishing.

Gorev, N. B., Gorev, V. N., Kodzhespirova, I. F., Shedlovsky, I. A. \& Sivakumar, P. (2018), 'Simulating control valves in water distribution systems as pipes of variable resistance', $J$. Water Resour. Plann. Manage. 144(11), 06018008.

Gorev, N. B., Kodzhespirov, I. F. \& Sivakumar, P. (2016), 'Non-unique steady states in water distribution networks with flow control valves', J. Hydraul. Eng. 142(9), 04016029.

IEEE (2008), 'IEEE standard 754-2008 for floating-point arithmetic', IEEE Standards Association. DOI: 10.1109/IEEESTD.2008.4610935.

Mathworks, T. (2016), MATLAB version 9.1.0.441655 (R2016b), Natick, Massachusetts. 
Piller, O. \& Bremond, B. (2001), Modeling of pressure regulating devices: A problem now solved, in 'World Water \& Environmental Resources congress, EWRI01', ASCE, Orlando, FL.

Piller, O. \& van Zyl, J. E. (2014), 'Modeling control valves in water distribution systems using a continuous state formulation', J. Hydraul. Eng. 140(11), 04014052.

Simpson, A. R. (1999), Modeling of pressure regulating devices a major problem yet to be satisfactorily solved in hydraulic simulation, ASCCE. Water Distribution Systems Conference.

Todini, E. \& Rossman, L. (2013), 'Unified framework for deriving simultaneous equation algorithms for water distribution networks.', J. Hydraul. Eng. 139(5), 511-526. 


\begin{tabular}{rrrrrrrrr} 
Pipe or node $i$ & $L_{i}(\mathrm{~m})$ & $D_{i}(\mathrm{~m})$ & $\epsilon_{i}(\mathrm{~mm})$ & $q_{\text {min }, i}(\mathrm{lps})$ & $q_{i}(\mathrm{lps})$ & $q_{\max , i}(\mathrm{lps})$ & $h_{i}(\mathrm{~m})$ & $\delta_{i}(\%)$ \\
\hline 1 & 1000 & 0.30 & 0.250 & $-\infty$ & 295.00 & $\infty$ & 193.3 & 100 \\
\hline 2 & 1000 & 0.20 & 0.250 & 0.00 & 110.00 & 110.00 & 99.5 & 0 \\
\hline 3 & 1500 & 0.15 & 0.250 & 1.00 & 10.00 & 10.00 & 108.9 & 67 \\
\hline 4 & 1200 & 0.20 & 0.250 & 2.00 & 50.00 & 50.00 & 100.0 & 4 \\
\hline 5 & 800 & 0.20 & 0.250 & 85.00 & 85.00 & 85.00 & 100.9 & 21 \\
\hline 6 & 800 & 0.15 & 0.250 & 12.00 & 12.00 & 89.00 & 101.2 & 24 \\
\hline 7 & 800 & 0.15 & 0.250 & 8.00 & 8.00 & 8.00 & 100.0 & 1 \\
\hline 8 & 1200 & 0.10 & 0.250 & -3.00 & -3.00 & -3.00 & 100.0 & 2 \\
\hline 9 & 1000 & 0.10 & 0.250 & 0.00 & 1.43 & 2.00 & - & - \\
\hline 10 & 800 & 0.10 & 0.250 & 0.00 & 1.57 & 5.00 & - & - \\
\hline
\end{tabular}

Table 1: The network parameters and the steady-state solution values for the illustrative network shown in Fig. 2. Also shown are the link flow constraints and the delivery fractions as percentages.

\begin{tabular}{rrrrrrrrrrrr}
$\mathrm{ID}$ & \multicolumn{1}{c}{$n_{p}$} & \multicolumn{1}{c}{$n_{j}$} & $n_{f}$ & $m$ & $\zeta(\%)$ & $\tau_{q}$ & $\tau_{h}$ & $\tau_{c}$ & $\rho_{e}$ & $\rho_{m}$ & $\rho_{o}$ \\
\hline$N_{1}$ & 934 & 848 & 8 & 11 & 82.4 & 14 & 16 & 17 & 17 & 6 & 11 \\
\hline$N_{2}$ & 1118 & 1039 & 2 & 11 & 54.2 & 11 & 13 & 15 & 17 & 7 & 12 \\
\hline$N_{3}$ & 1976 & 1770 & 4 & 12 & 93.3 & 11 & 16 & 17 & 17 & 8 & 9 \\
\hline$N_{4}$ & 2465 & 1890 & 3 & 13 & 23.3 & 11 & 12 & 15 & 16 & 12 & 15 \\
\hline$N_{5}$ & 2508 & 2443 & 2 & 12 & 28.4 & 12 & 16 & 18 & 17 & 5 & 9 \\
\hline$N_{6}$ & 8584 & 8392 & 2 & 10 & 60.8 & 11 & 16 & 18 & 17 & 8 & 12 \\
\hline$N_{7}$ & 14830 & 12523 & 7 & 13 & 47.5 & 12 & 14 & 15 & 16 & 10 & 14 \\
\hline$N_{8}$ & 19647 & 17971 & 15 & 10 & 97.6 & 10 & 14 & 18 & 16 & 8 & 11 \\
\hline
\end{tabular}

Table 2: Convergence results for the ASMFC applied to the $N_{1}-N_{8}$ case study networks with 60 cotree links flow-constrained, 3 of which have LFEC, and demand magnification factor $f=5$. 


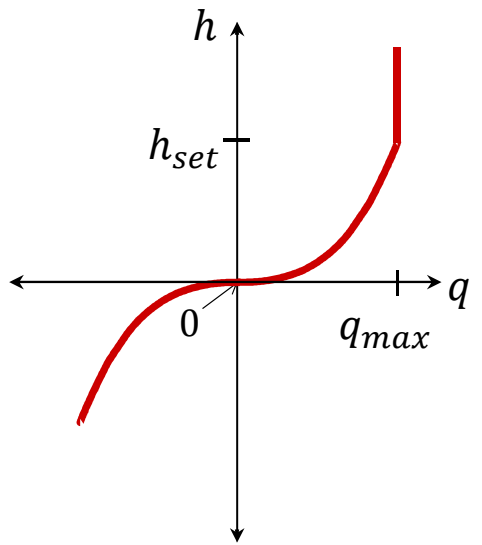

Figure 1: The multivalued subdifferential mapping modelling an FCV. 


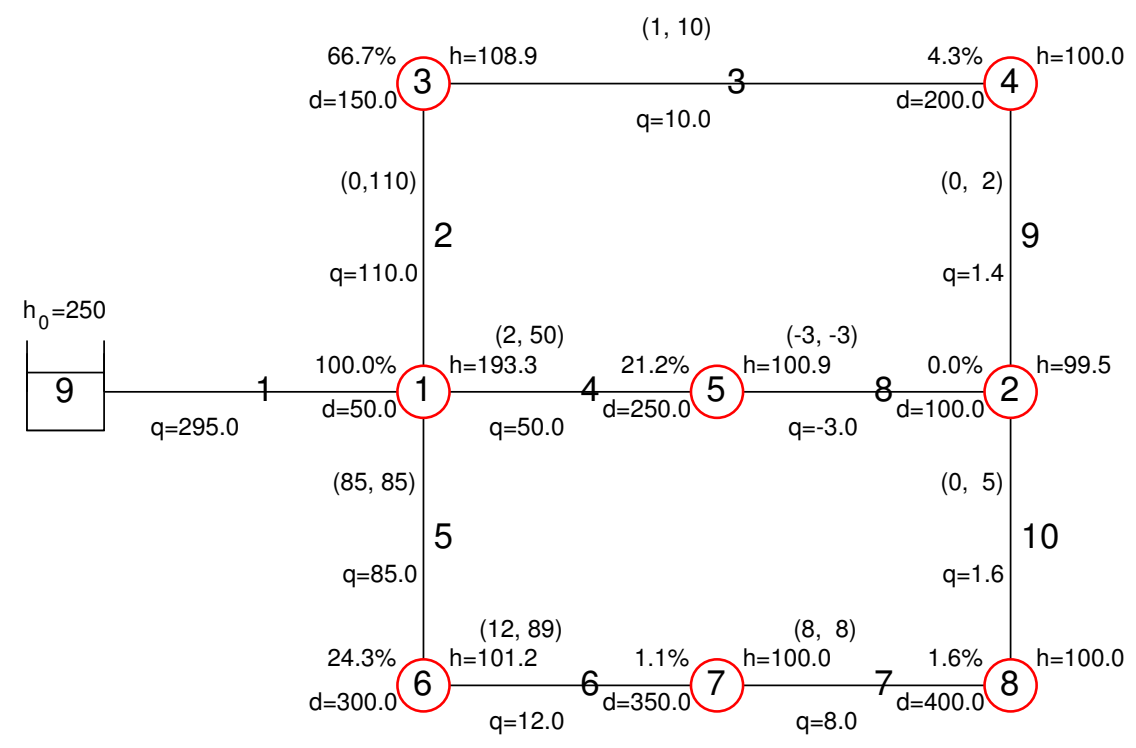

Figure 2: The illustrative network discussed in the text showing the steady-state solution and the link flow constraints. 


\section{${ }_{711}$ List of Figures}

${ }_{712} \quad 1$ The multivalued subdifferential mapping modelling an FCV. . . . . . . . . . . . 30

${ }_{713} 2$ The illustrative network discussed in the text showing the steady-state solution

714 and the link flow constraints. . . . . . . . . . . . . . . . . . . 31 


\section{${ }_{715}$ List of Tables}

716 1 The network parameters and the steady-state solution values for the illustrative network shown in Fig. 2. Also shown are the link flow constraints and the

2 Convergence results for the ASMFC applied to the $N_{1}-N_{8}$ case study network$\mathrm{s}$ with 60 cotree links flow-constrained, 3 of which have LFEC, and demand magnification factor $f=5 . \ldots \ldots \ldots$. . . . . . . . . . . . . 29 